\title{
The Behavior of Molecular Measures of Natural Selection after a Change in Population Size
}

\author{
Rebekka Müller*, Ingemar Kaj*, Carina F. Mugal ${ }^{\dagger, 1}$ \\ * Department of Mathematics, Uppsala University, 75237 Uppsala, Sweden \\ ${ }^{\dagger}$ Department of Ecology and Genetics, Uppsala University, 75236 Uppsala, Sweden \\ ${ }^{1}$ Norbyvägen 18D, 752 36 Uppsala, Sweden
}




\section{Short running title}

8 Measures of natural selection

\section{Keywords}

10 theoretical population genetics; nearly neutral theory; natural selection; ge-

11 netic drift; effective population size

${ }_{12}$ Corresponding author

13 Carina F. Mugal

14 Office mailing address:

15 Evolutionsbiologiskt centrum, Norbyvägen 18D, 75236 Uppsala, Sweden

16 Phone number: +4618-471 6466

17 Email address: Carina.Mugal@ebc.uu.se 
A common model to describe natural selection at the molecular level is

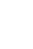 \\ ABSTRACT}

he nearly neutral theory, which emphasizes the importance of mutations with slightly deleterious fitness effects as they have a chance to get fixed due to genetic drift. Since genetic drift is stronger in smaller than in larger populations, a negative relationship between molecular measures of selection and population size is expected within the nearly neutral theory. Originally, this hypothesis was formulated under equilibrium conditions. A change in population size, however, pushes the selection-drift balance off equilibrium leading to alterations in the efficacy of selection. To investigate the nonequilibrium behavior, we relate measures of natural selection and genetic drift to each other, considering both, measures of micro- and macroevolution. Specifically, we use a Poisson random field framework to model $\pi_{N} / \pi_{S}$ and $\omega$ as time-dependent measures of selection and assess genetic drift by an effective population size. This analysis reveals a clear deviation from the expected equilibrium selection-drift balance during nonequilibrium periods. Moreover, we find that microevolutionary measures quickly react to a change in population size and reflect a recent change well, at the same time as they quickly lose the knowledge about it. Macroevolutionary measures, on the other hand, react more slowly to a change in population size but instead capture the influence of ancient changes longer. We therefore conclude that it is important to be aware of the different behaviors of micro- and macroevolutionary measures when making inference in empirical studies, in particular when comparing results between studies. 


\section{INTRODUCTION}

Among the key driving factors of evolution are mutations, selection, and genetic drift. The analysis of the interplay between them therefore provides valuable understanding on a population's ability to evolve and adapt. Population genetics theory predicts that the strength of genetic drift is weaker in larger populations than in smaller populations, due to the stochastic nature of reproduction (Wright 1931; Kimura 1964). This results in a positive correlation between the efficacy of selection and population size, and hence deleterious mutations are more likely to go extinct due to purifying selection if population size is large. This relationship can for example explain why asexual (Lynch et al. 1993; Lumley et al. 2015), small, or endangered populations (Lynch et al. 1995; Lachapelle et al. 2017) have a higher risk to go extinct or suffer from genetic diseases (Karlsson et al. 2014; Seppälä 2015).

The relationship between the efficacy of selection and population size also provides the foundation for the nearly neutral theory of molecular evolution (Ohta 1973, 1976, 1992). In this framework, typically the distribution of fitness effects (DFE) of new mutations is weighted towards purifying selection: most mutations are deleterious, of which a nonnegligible amount is slightly deleterious, and only a small proportion of mutations is advantageous. While strongly deleterious mutations rarely segregate in the population or get fixed, slightly deleterious mutants contribute significantly to segregating polymorphisms and can eventually get fixed due to genetic drift. The fact that only a small fraction of mutants is under positive selection makes the nearly neutral theory predict a negative correlation between molecular measures of natural selection and population size. The validity of this hypothesis can be tested 
by comparing measures of selection and genetic drift, and has been supported across a wide range of taxa (e.g. Hughes 2008; Elyashiv et al. 2010; Figuet et al. 2016; Chen et al. 2017; Bolívar et al. 2019).

The traditional approach to assessing genetic drift is to apply some concept of an effective population size, $N_{\text {eff. }}$. The concept of $N_{\text {eff }}$ is used to compare a given (non-ideal) population with a simpler idealized reference model, such as the ideal Wright-Fisher model, with respect to a particular property. This leads to different definitions of $N_{\text {eff }}$, e.g. inbreeding and variance (Wright 1931; Crow and Kimura 1970) or eigenvalue effective size (Ewens 1979). All approaches predict $N_{\text {eff }}$ under different circumstances as for example certain spatial and temporal scales and demographic scenarios. Most often it is not evident whether underlying assumptions of the various models are met and how accurate resulting estimates of $N_{\text {eff }}$ are when assumptions are violated. For this, the spatial and temporal scales of different estimates of $N_{\text {eff }}$ have to be interpreted (Wang et al. 2016) to draw the right conclusions. These issues are discussed frequently in the literature (Ewens 1969; Sjödin et al. 2004; Waples 2005; Wang et al. 2016; Galtier and Rousselle 2020) confirming the need for more clarification in this field.

To detect evidence of selection in genome data, different approaches and methods have been developed. A common feature of them is to contrast neutral reference and test data. Under the assumption that synonymous mutations are neutral, the contrast between synonymous and nonsynonymous mutations in protein-coding sequences provides a relevant approach. Measures of natural selection at the microevolutionary timescale, which are based on polymorphisms, are designed to identify selective events within a 
species and give a snapshot of the current state. A popular representative is the ratio of nonsynonymous and synonymous diversity, $\pi_{N} / \pi_{S}$ (Nei and Li 1979). Other measures look at interspecific differences in form of fixations that arise through events which happened in a lineage since a speciation event. Those measures are accumulative and give insight into the macroevolution of a population or species. A measure that belongs to this group is the ratio of the nonsynonymous and synonymous fixation rate, $\omega$, commonly assessed by the ratio of nonsynonymous and synonymous sequence divergence (Goldman and Yang 1994; Muse and Gaut 1994).

The nearly neutral theory predicts a negative correlation between $N_{\text {eff }}$ and the measures $\pi_{N} / \pi_{S}$ and $\omega$ : the smaller $N_{\text {eff }}$ the larger the ratios, i.e. the less efficient selection. However, the prediction is based on the equilibrium assumption, where the effect of genetic drift on segregating sites balances the efficacy of selection in a stable manner implying a constant evolutionary rate. Yet there are many factors that can cause a nonequilibrium such as a change in population size, population structure or migration, a change in recombination rate, or environmental variation (Brandvain and Wright 2016). These events disturb the selection-drift balance.

Several empirical studies (Lu et al. 2006; Campos et al. 2014; Hollister et al. 2014; Renaut and Rieseberg 2015; Leroy et al. 2020; Galtier and Rousselle 2020) provide evidence that the efficacy of natural selection is limited in nonequilibrium conditions. In a meta-analysis, Brandvain and Wright (2016) compare predictions of classical theory with results from a large number of data analyses, which stresses the need of care for nonequilibrium conditions when testing for differences in selection efficacy between species. To enable 
such care to be taken, theoretical models are a critical tool to provide conceptual understanding of the interplay between selection and genetic drift and the extent of nonequilibrium periods. Given the mathematical challenges in modeling natural selection in nonequilibrium scenarios, such studies are still rare. Gordo and Dionisio (2005), for example, develop a nonequilibrium model to estimate parameters of deleterious mutations, which can be applied to quantify the mutation rate to deleterious alleles. Alternatively, simulations can be used to provide observational insight into the nonequilibrium behavior of measures of evolution. Rousselle et al. (2018) investigate the rate of adaptive molecular evolution in fluctuating population size and find that this violation of the equilibrium assumption can lead to a severely biased inference based on current methodology.

In this study we investigate the effect of a change in population size on micro- and macroevolutionary measures of selection in an otherwise ideal population. While being aware of other factors that lead to a nonequilibrium, concentrating on this isolated aspect provides conceptual understanding that is straight forward to interpreted. For this purpose, we first introduce a mathematical model and state and derive relevant quantities needed to model measures of selection in nonequilibrium. Among these, an important statistic is the allele frequency spectrum (AFS) from which other measures such as nucleotide diversity can be derived. Several different approaches exist to model the AFS in nonequilibrium (Evans et al. 2007; Živković and Stephan 2011; Živković et al. 2015; Kaj and Mugal 2016). We here build on the Poisson random field framework approach as in Kaj and Mugal (2016). With the help of this framework, the above addressed measures, $\pi_{N} / \pi_{S}$ and $\omega$, 
are formulated as functions of time, $\left(\pi_{N} / \pi_{S}\right)(t)$ and $\omega(t)$. Equipped with the analytical model, we discuss the following questions. First, how does a change in population size affect micro- and macroevolutionary measures of natural selection, $\left(\pi_{N} / \pi_{S}\right)(t)$ and $\omega(t)$ ? Second, does the prediction of the nearly neutral theory also hold in a nonequilibrium? To this end we investigate different choices of $N_{\text {eff }}$ as measures of genetic drift. Finally, we discuss the relevance of micro- and macroevolutionary measures in the study of natural selection.

\section{MODELS AND METHODS}

\section{Model formulation}

Let $N$ represent the population size of a haploid population-which under the assumption of additive fitness effects in a diploid organism, is equivalent to a diploid population of size $N / 2$. We consider the evolution of a population that undergoes an instantaneous change in population size at a single point in time $t^{*}$ from constant size $N$ to constant size $\kappa N$, where $\kappa$ is a positive parameter. In other words, the population size over time is a step function $N_{\kappa}$ such that $N_{\kappa}(t)=N, t<t^{*}$, and $N_{\kappa}(t)=\kappa N, t \geq t^{*}$. Throughout this work we use $N$ as reference size and apply an evolutionary timescale where $t$ units of time correspond to $[N t]$ generations. Each individual is characterized by $L$ independent sites, which corresponds to the assumption of free recombination between sites. Random mutations arrive independently and uniformly over individuals on mono-allelic sites with population mutation intensity $\theta$ per generation in the reference population. Hence, as long as $t \leq t^{*}$, the mutation intensity per time unit is $\theta N$. Consequently, for $t \geq t^{*}$, 
As $N \rightarrow \infty$, the scaled fixation rate emerges as

$$
N q_{\gamma, \kappa}(1 / N) \rightarrow \omega_{\gamma, \kappa}=\frac{2 \gamma \kappa}{1-e^{-2 \gamma \kappa}}, \quad \gamma \neq 0, \quad \omega_{0, \kappa}=1 .
$$




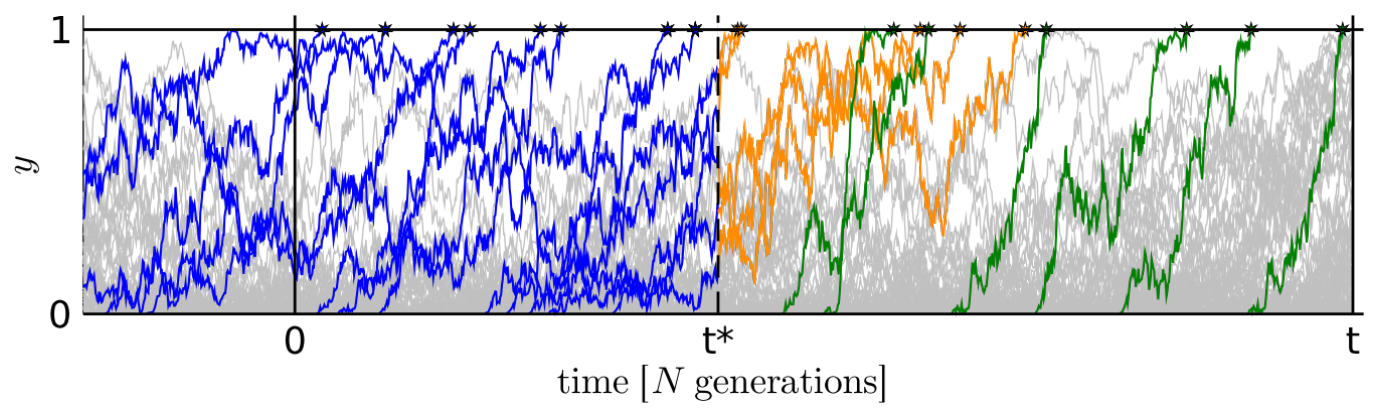

Figure 1: Polymorphisms and fixations in $[0, t]$. Vertical lines as for example at $t^{*}$ represent a snapshot of the population dynamics and the distribution of allele frequencies at that specific point in time are summarized in the AFS. Polymorphisms in grey go to extinction or still segregate at $t$. Polymorphisms leading to a substitution are colored: fixations before $t^{*}$ (blue), fixations after $t^{*}$ emerging from segregrating polymorphisms at $t^{*}$ (orange), and fixations of mutations that arose after the change in population size (green).

This means, in an equilibrium population of size $\kappa N$, the fixation rate ratio of a class of selected (with selective pressure $\gamma$ ) and neutral mutations in the limit equals $\omega_{\gamma, \kappa}$.

Returning to the Poisson random field setting, the allele frequencies are represented by Poisson points $\left(s, \xi^{s}\right)$ on the collection of sites according to the Poisson distribution with intensity $\theta N_{\kappa}$ : once such a mutation event takes place at a certain time $s$, a path $\left(\xi_{t}^{s}\right)_{t \geq s}$ is initialized at frequency $1 / N_{\kappa}(s)$. We fix $t^{*}>0$ and represent the state of the Poisson random field at time $t$ as a random measure $\mathcal{X}_{t}^{N_{\kappa}}(d y)$ on $(0,1]$. For $t \leq 0$, however, we will ignore fixations but start from polymorphic frequencies on $(0,1)$ at $t=0$. A visualization of the setup is presented in Fig. 1. It is known that the aggregate of all mutations from the infinite past in the ancestral population 
together build up a Poisson measure in steady state. Let

$$
\psi_{\gamma, \kappa}(y)=\frac{1-e^{-2 \gamma \kappa(1-y)}}{\gamma y(1-y)}, \quad \gamma \neq 0, \quad \psi_{0, \kappa}(y)=\frac{2 \kappa}{y} .
$$

Now the relevant initial distribution at $t=0$ for our model, that is $\mathcal{X}_{0}^{N_{\kappa}}(d y)$, is a Poisson measure with intensity measure $\omega_{\gamma, 1} \psi_{\gamma, 1}(y) d y$ on $(0,1)$. The initial distribution at $t=0$ plus the arrival of new mutations during $(0, t]$ together preserve the Poisson distribution which is invariant as long as the population size does not change, i.e. for $0<t \leq t^{*}$. To account for fixations during $[0, t]$ we also include the singular contribution at $y=1, \mathcal{X}_{t}^{N_{\kappa}}(\{1\})$, $t \geq 0$, which is a Poisson counting process with time-inhomogeneous intensity. Formally, we construct our model $\mathcal{X}_{t}^{N_{\kappa}}$ using stochastic Poisson integrals. The detailed presentation and most of the technical aspects are deferred to the appendix sections. For $0<t \leq t^{*}$,

$$
\begin{aligned}
\left\langle\mathcal{X}_{t}^{N_{\kappa}}, f\right\rangle= & \int_{0}^{1} \int_{\mathcal{D}} f\left(\xi_{t}^{0, y}\right) \mathcal{M}_{\gamma}\left(d y, d \xi^{0, y}\right) \\
& +\int_{0}^{t} \int_{0}^{1} \int_{\mathcal{D}} f\left(\xi_{t}^{s, y}\right) \mathcal{N}_{N_{\kappa}}\left(d s, d y, d \xi^{s, y}\right),
\end{aligned}
$$

for suitable functions $f \in \widetilde{\mathcal{F}}$, specified in Appendix A, satisfying sufficient conditions for these integrals to be well defined. In particular, $f(0)=0$. The class $\mathcal{D}$ is the path space for the diffusion processes $t \mapsto \xi_{t}$, consisting of functions $g: \mathbb{R} \rightarrow[0,1]$ which are right continuous and have left limits. Moreover, $\mathcal{M}_{\gamma}\left(d y, d \xi^{0, y}\right)$ is a Poisson random measure on $[0,1] \times \mathcal{D}$ with intensity $m_{\gamma, 1}\left(d y, d \xi^{0, y}\right)=\theta \omega_{\gamma, 1} \psi_{\gamma, 1}(y) d y \mathbb{P}_{y}^{\gamma, 1}\left(d \xi^{0, y}\right)$ and $\mathcal{N}_{N_{\kappa}}\left(d s, d y, d \xi^{s, y}\right)$ is a Poisson random measure on $\mathbb{R} \times[0,1] \times \mathcal{D}$ with intensity measure $n_{N_{\kappa}}\left(d s, d y, d \xi^{s, y}\right)=$ $\theta N_{\kappa}(s) d s \delta_{1 / N_{\kappa}(s)}(d y) \mathbb{P}_{y}^{\gamma, N_{\kappa} / N}\left(d \xi^{s, y}\right)$. The first term in Eq. (5) represents the family of ancestral allele frequencies with initial values at $t=0$ given by 
the Poisson measure $\mathcal{X}_{0}^{N_{\kappa}}(d y), 0<y<1$. The second term contains additional allele frequencies sparked off by mutations during $(0, t]$. Similarly, the collection of paths at some time $t>t^{*}$ consists of two contributions,

$$
\begin{aligned}
\left\langle\mathcal{X}_{t}^{N_{\kappa}}, f\right\rangle= & \int_{0}^{1} \int_{\mathcal{D}} f\left(\xi_{t}^{t^{*}, y}\right) \mathcal{M}_{\gamma, \kappa}^{\mathcal{X}_{t^{*}}}\left(d y, d \xi^{t^{*}, y}\right) \\
& +\int_{t^{*}}^{t} \int_{0}^{1} \int_{\mathcal{D}} f\left(\xi_{t}^{s, y}\right) \mathcal{N}_{N_{\kappa}}\left(d s, d y, d \xi^{s, y}\right) .
\end{aligned}
$$

Here, conditional on $\mathcal{X}_{t^{*}}, \mathcal{M}_{\gamma, \kappa}^{\mathcal{X}_{t^{*}}}\left(d y, d \xi^{*}, y\right)$ is a Poisson random measure on $(0,1] \times \mathcal{D}$ with intensity $\mathcal{X}_{t^{*}}^{N_{\kappa}}(d y) \mathbb{P}_{y}^{\gamma, \kappa}\left(d \xi^{*}, y\right)$. This term represents the fate of the allele frequencies extending beyond $t^{*}$ of all alleles, polymorphic or fixed, which were present at $t^{*}$. The additional term again covers new mutations taking place subsequent to the change in population size.

\section{The nonequilibrium AFS}

The AFS accounts for the collection of all derived allele frequencies across sites at a fixed point in time. The spectrum of allele frequencies $y, 0<y<1$, represents the average intensity of attained frequency values at $t, \xi_{t}^{s}=y$ for some $s \leq t$, compare Fig. 1. In our approach we also include alleles which have reached fixation during $[0, t]$. As a reference case we begin with the equilibrium AFS, which arises as the scaled limit of expected values for the case of a fixed size population, say $\kappa N$. Then,

$$
\lim _{N \rightarrow \infty} \mathbb{E}\left\langle\mathcal{X}_{t}^{\kappa N}, f\right\rangle=\theta \omega_{\gamma, \kappa} f(1) t+\theta \int_{0}^{1} f(y) \omega_{\gamma, \kappa} \psi_{\gamma, \kappa}(y) d y,
$$

see Lemma 3 iii) in Appendix A. In particular, taking $\kappa=1$, the limiting expected value of relation (5) is given by this linear function, as long as $t \leq t^{*}$. The linear term in $t$ represents the effect of constant rate fixations and the 
integral term independent of time represents the steady-state spectrum of polymorphic frequencies.

In order to analyze the nonequilibrium AFS caused by applying population size $N_{\kappa}$, we derive the limiting expected values of each term in Eq. (6), see Theorem 1, Appendix B. The limiting expected contribution from the ancestral component in Eq. (6), mutations which occurred prior to $t^{*}$, equals

$$
\begin{aligned}
\lim _{N \rightarrow \infty} & \mathbb{E}\left[\int_{0}^{1} \int_{\mathcal{D}} f\left(\xi_{t}^{t^{*}, y}\right) \mathcal{M}_{\gamma, \kappa}^{\mathcal{X}_{t^{*}}}\left(d y, d \xi^{t^{*}, y}\right)\right] \\
& =\theta \omega_{\gamma, 1} f(1) t^{*}+\theta \int_{0}^{1} \mathbb{E}_{y}^{\gamma, \kappa}\left[f\left(\xi_{t}^{t^{*}}\right)\right] \omega_{\gamma, 1} \psi_{\gamma, 1}(y) d y
\end{aligned}
$$

compare Eq. (B.1) in Theorem 1. Similarly, the limiting expected value of the latter contribution in Eq. (6) yields the nonstationary build-up AFS (Kaj and Mugal 2016, Theorem 1), that arises from a completely mono-allelic population. In Lemma 3 i) together with Theorem 1 we show

$$
\begin{aligned}
\lim _{N \rightarrow \infty} & \mathbb{E} \\
& \left.=\theta \int_{t^{*}}^{t} \int_{0}^{1} \int_{\mathcal{D}} f\left(\xi_{t}^{s, y}\right) \mathcal{N}_{N_{\kappa}}\left(d s, d y, d \xi^{s, y}\right)\right] \\
& \theta \omega_{\gamma, \kappa} f(1)\left(t-t^{*}\right)+\theta \int_{0}^{1}\left(f(y)-\mathbb{E}_{y}^{\gamma, \kappa}\left[f\left(\xi_{t}^{t^{*}}\right)\right]\right) \omega_{\gamma, \kappa} \psi_{\gamma, \kappa}(y) d y
\end{aligned}
$$

While in these representations we do not see directly a spectrum of frequencies $y$ with explicit weights affecting $f(y)$, we do see indirectly the timedependence effect due to the nonequilibrium framework. By adding up both contributions we obtain our main result regarding the nonequilibrium AFS, 


$$
\begin{aligned}
\lim _{N \rightarrow \infty} & \mathbb{E}\left\langle\mathcal{X}_{t}^{N_{\kappa}}, f\right\rangle \\
& =\theta f(1)\left\{\omega_{\gamma, 1} t^{*}+\omega_{\gamma, \kappa}\left(t-t^{*}\right)\right\}+\theta \int_{0}^{1} f(y) \omega_{\gamma, \kappa} \psi_{\gamma, \kappa}(y) d y \\
& +\theta \int_{0}^{1} \mathbb{E}_{y}^{\gamma, \kappa} f\left(\xi_{t}^{t^{*}}\right)\left(\omega_{\gamma, 1} \psi_{\gamma, 1}(y)-\omega_{\gamma, \kappa} \psi_{\gamma, \kappa}(y)\right) d y,
\end{aligned}
$$

see Theorem 1 in Appendix B for a detailed derivation.

We make two remarks: for $f \in \widetilde{\mathcal{F}}$ with the additional property $f(1)=0$, the time-dependent AFS provides a bridge between the "marginal" limit spectra

$\mathbb{E}\left\langle\mathcal{X}_{t^{*}}^{N_{\kappa}}, f\right\rangle \rightarrow \theta \int_{0}^{1} f(y) \omega_{\gamma, 1} \psi_{\gamma, 1}(y) d y, \mathbb{E}\left\langle\mathcal{X}_{\infty}^{N_{\kappa}}, f\right\rangle \rightarrow \theta \int_{0}^{1} f(y) \omega_{\gamma, \kappa} \psi_{\gamma, \kappa}(y) d y$

For the case of neutral evolution, $\gamma=0$, we have $\omega_{0,1} \psi_{0,1}(y)-\omega_{0, \kappa} \psi_{0, \kappa}(y)=$ $2(1-\kappa) / y$ and hence, for $f \in \widetilde{\mathcal{F}}, t \geq t^{*}$, and $N \rightarrow \infty$, $\mathbb{E}\left\langle\mathcal{X}_{t}^{N_{\kappa}}, f\right\rangle \rightarrow 2 \theta(1-\kappa) \int_{0}^{1} y^{-1} \mathbb{E}_{y}^{0, \kappa}\left[f\left(\xi_{t}^{t^{*}}\right)\right] d y+2 \theta \kappa \int_{0}^{1} y^{-1} f(y) d y+\theta f(1) t$.

Application of selected functions $f$ to the nonequilibrium AFS in Eq. (10) allows to retrieve expressions for relevant summary statistics, such as nucleotide diversity and the fixation rate.

\section{The ratio of nucleotide diversity during nonequilibrium}

We derive and investigate the ratio of nucleotide diversity, $\pi_{N} / \pi_{S}$ (Nei and Li 1979), in a population undergoing a change in population size according to $N_{\kappa}$. Nucleotide diversity measures the number of pairwise differences, which entails integrating the specific function $f_{\mathrm{pw}}(y)=2 y(1-y)$ with respect to the AFS. As a reference case we observe that during equilibrium in a population 
controlled by a size parameter $\kappa$ and a fixed selection coefficient $\gamma \neq 0$, we have by Eq. (7) with $f_{\mathrm{pw}}(1)=0$,

$$
\pi_{N}^{\gamma, \kappa}=\theta \int_{0}^{1} 2 y(1-y) \omega_{\gamma, \kappa} \psi_{\gamma, \kappa}(y) d y=4 \theta \kappa\left(\frac{1}{1-e^{-2 \gamma \kappa}}-\frac{1}{2 \gamma \kappa}\right) .
$$

More generally, by applying Eq. (10), we obtain the time-dependent nonsynonymous nucleotide diversity measure $\pi_{N}^{\gamma, \kappa}(t)=\lim _{N \rightarrow \infty} \mathbb{E}\left\langle\mathcal{X}_{t}^{N_{\kappa}}, f_{\mathrm{pw}}\right\rangle$ in nonequilibrium.

In order to allow for variation in selection across sites for the nonsynonymous diversity, we integrate the previous expressions over a distribution of fitness effects (DFE). We will denote the random variable generating the $\gamma$-values by $\mathcal{V}$ and assume it has a continuous density function $h_{\mathcal{V}}$. Because of the presumed rarity or negligibility of advantageous mutations at the genome-wide level within the nearly neutral theory, we focus on weak and strong purifying selection following Eyre-Walker et al. (2006); Loewe and Charlesworth (2006); Galtier and Rousselle (2020). A common choice of DFE in this scenario is the negative gamma distribution. The density function is

$$
h_{\mathcal{V}}(v)=\frac{(-v)^{a-1} e^{v / b}}{b^{a} \Gamma(a)}, \quad v \leq 0,
$$

with shape parameter $a>0$, scale parameter $b>0$, and mean $-a b$. Integration of the expression in Eq. (11) and $\pi_{N}^{\gamma, \kappa}(t)$ over this density yields an averaged diversity measure $\pi_{N}^{\kappa}$. Taken together it holds

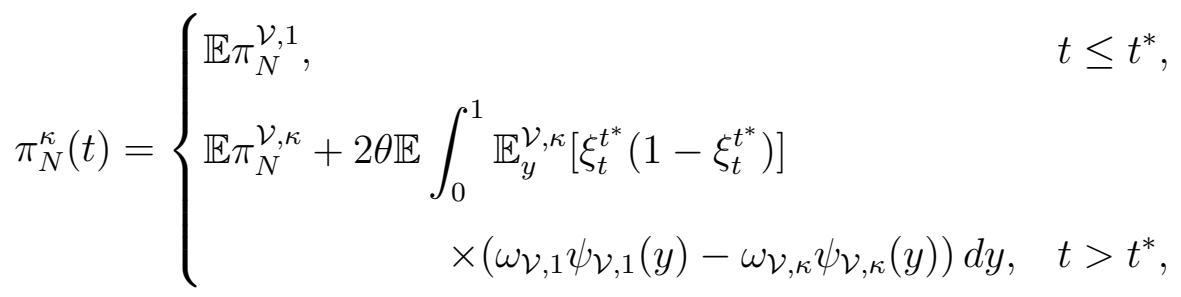


see Appendix C for details. The expectations in the above expression are used to indicate integration over the DFE. We observe that $\pi_{N}^{\kappa}(t)$ approaches a new equilibrium, $\pi_{N}^{\kappa}(t) \rightarrow \mathbb{E} \pi_{N}^{\mathcal{V}, \kappa}$ as $t \rightarrow \infty$, since $\xi_{t}^{t^{*}} \in\{0,1\}$ for $t \rightarrow \infty$. For the case of neutral evolution, $\gamma=0$, the result simplifies considerably and we obtain the synonymous diversity as

$$
\pi_{S}^{\kappa}(t)= \begin{cases}2 \theta, & t \leq t^{*}, \\ 2 \theta \kappa+2 \theta(1-\kappa) e^{-\left(t-t^{*}\right) / \kappa}, & t>t^{*}\end{cases}
$$

with $\pi_{S}^{\kappa}(t) \rightarrow 2 \theta \kappa$ as $t \rightarrow \infty$. The ratio of nonsynonymous and synonymous diversity, which we denote $\left(\pi_{N} / \pi_{S}\right)^{\kappa}(t):=\pi_{N}^{\kappa}(t) / \pi_{S}^{\kappa}(t)$, is also time dependent and determined by Eqs. (13) and (14). The ratio in equilibrium will be denoted $\left(\pi_{N} / \pi_{S}\right)^{\kappa}$.

\section{The fixation rate ratio during nonequilibrium}

The nonsynonymous to synonymous fixation rate ratio of selected and neutral mutations (Goldman and Yang 1994; Muse and Gaut 1994), $\omega^{\kappa}(t)$, is determined by the ratio of the number of nonsynonymous and synonymous fixations in a finite interval. We therefore consider the number of fixations in the population during $[0, t]$ with a change in population size as before given by $N_{\kappa}$. To account for fixations in the random field setting, we wish to count all Poisson points $\left(s, \xi^{s}\right)$ such that $\xi_{t}^{s}=1$. In other words, we evaluate the indicator function $f_{\text {fix }}(y):=\mathbb{1}_{\{1\}}(y)$ at the nonequilibrium AFS, Eq. (10). Hence, the number of fixations in the population during $[0, t]$ with a change in population size given by $N_{\kappa}$ is $Z^{\gamma, \kappa}(t):=\lim _{N \rightarrow \infty} \mathbb{E}\left\langle\mathcal{X}_{t}^{N_{\kappa}}, f_{\text {fix }}\right\rangle$. The decomposition of $\lim _{N \rightarrow \infty} \mathbb{E}\left\langle\mathcal{X}_{t}^{N_{\kappa}}, f_{\text {fix }}\right\rangle$ into the ancestral contribution in Eq. (8) and the buildup in Eq. (9) allows for matching the different categories 
of fixations in Fig. 1 with the corresponding analytic representation: the first term in Eq. (8) reflects fixations (in blue) appearing during $\left[0, t^{*}\right]$, whereas the second part corresponds to fixations (in orange) during $\left[t^{*}, t\right]$ for which the mutation happened before $t^{*}$. The buildup component in Eq. (9) accounts for fixations (in green) during $\left[t^{*}, t\right]$ for which the mutation occurred after $t^{*}$.

To obtain an explicit representation of $Z^{\gamma, \kappa}(t)$, we note that $f_{\text {fix }}(1)=1$ and that the expectation operator applied to $f_{\text {fix }}(y)$ can be rewritten in terms of the fixation time distribution,

$$
\mathbb{E}_{y}^{\gamma, \kappa}\left[f_{\text {fix }}\left(\xi_{t}\right)\right]=\mathbb{E}_{y}^{\gamma, \kappa}\left[\mathbb{1}_{\{1\}}\left(\xi_{t}\right)\right]=\mathbb{P}_{y}^{\gamma, \kappa}\left(\xi_{t}=1\right)=\mathbb{P}_{y}^{\gamma, \kappa}\left(\tau_{1} \leq t\right)
$$

Thus,

$$
Z^{\gamma, \kappa}(t)=\left\{\begin{array}{lr}
\theta \omega_{\gamma, 1} t, & t \leq t^{*}, \\
\theta\left\{\omega_{\gamma, 1} t^{*}+\omega_{\gamma, \kappa}\left(t-t^{*}\right)\right\} & \\
\quad+\theta \int_{0}^{1} \mathbb{P}_{y}^{\gamma, \kappa}\left(\tau_{1} \leq t-t^{*}\right)\left(\omega_{\gamma, 1} \psi_{\gamma, 1}(y)-\omega_{\gamma, \kappa} \psi_{\gamma, \kappa}(y)\right) d y, & t>t^{*}
\end{array}\right.
$$

compare Appendix D for technical details. Fixations that originate from nonsynonymous mutations are averaged over the DFE in Eq. (12); for synonymous fixations $\gamma$ is set to zero. Finally, the fixation rate ratio in nonequilibrium is defined as

$$
\omega^{\kappa}(t)=\frac{\mathbb{E} Z^{\mathcal{V}, \kappa}(t)}{Z^{0, \kappa}(t)} .
$$

The nonequilibrium quantity $\omega^{\kappa}(t)$ is consistent with the equilibrium fixation rate ratio $\omega_{\gamma, \kappa}$ stated in Eq. (3), since $\omega^{\kappa}(t)=\mathbb{E} \omega_{\mathcal{V}, 1}$ for $t \leq t^{*}$ and $\omega^{\kappa}(t) \rightarrow$ $\mathbb{E} \omega_{\mathcal{V}, \kappa}$ for $t \rightarrow \infty$.

We note that measures modeled in this study are population functionalsin contrast to sample functionals which can be viewed as estimators of pop- 
ulation functionals. In case of $\left(\pi_{N} / \pi_{S}\right)^{\kappa}(t)$ the population and sample functional are equal. When using nonsynonymous over synonymous sequence divergence as estimate for the fixation rate ratio $\omega^{\kappa}(t)$, sample functional and population functional are not equal but converge for $t \rightarrow \infty$ (Wolf et al. 2009; Mugal et al. 2014, 2020).

\section{Stochastic simulations}

For performing stochastic simulation of paths $\left(\xi_{t}\right)_{t}$, we apply the discrete Wright-Fisher model with selection to a population of size $N$. It suffices to simulate paths for the reference population, since a polymorphism in a population of size $\kappa N$ evolves as in the reference population but with time scaled by $\kappa$. Paths are simulated over a maximum of $n$ generations using the binomial Wright-Fisher sampling with selection. The selection coefficient in the discrete setting is obtained from the relation $s=\gamma / N$.

For the distribution of the time to fixation, $\mathbb{P}_{y}^{\gamma, \kappa}\left(\tau_{1} \leq t\right)$, we generate $m_{\tau_{1}}$ paths for each tuple $(y, \gamma)$. If the derived allele does not get fixed, the time to fixation is set to infinity. Otherwise the fixation time is set to the generation it got fixed. Finally, the distribution function of the time to fixation on the evolutionary timescale is obtained by scaling generations with $N$. For the expected value $\mathbb{E}_{y}^{\gamma, \kappa}\left[f\left(\xi_{t}^{t^{*}}\right)\right]$ in the nonequilibrium AFS we simulate and average over $m_{\xi}$ paths for each triplet $\left(y, \gamma,\left(t-t^{*}\right) / \kappa\right)$.

Parameters that we used are $N=1000, n=20.000, s \in[-1,0]$ or equivalently $\gamma \in[-1000,0], m_{\tau_{1}}=100.000$ (if $\gamma=0$ ) and $m_{\tau_{1}}=10.000$ (if $\gamma<0$ ), respectively, $m_{\xi}=1000$. 


\section{Data availability statement}

Code used to implement the stochastic simulations can be found on GitLab.

\section{RESULTS AND DISCUSSION}

\section{Measures of natural selection in a nonequilibrium population}

We study the behavior of measures of natural selection as functions of time after a change in population size. Figure $2 \mathrm{~A}$ shows the behavior of $\left(\pi_{N} / \pi_{S}\right)^{\kappa}(t)$ for $\kappa \in\{0.1,0.25,0.5,1,2,4\}$. The time it takes to reach the new equilibrium depends on the direction of change in population size: the new equilibrium is reached quickly in case of a reduction $(\kappa<1)$, whereas for an increase in size $(\kappa>1)$ it takes longer. Also the extent of change determines how fast $\left(\pi_{N} / \pi_{S}\right)^{\kappa}(t)$ attains the new equilibrium value. The more the population size is reduced, the faster $\left(\pi_{N} / \pi_{S}\right)^{\kappa}(t)$ reaches its new equilibrium value; the more a population increases in size, the longer it takes. Also, given a DFE restricted to deleterious mutations, $\left(\pi_{N} / \pi_{S}\right)^{\kappa}(t)$ is negatively correlated with population size as predicted by the nearly neutral theory of molecular evolution.

The behavior of $\omega^{\kappa}(t)$ after a change in population size is depicted in Fig. 2B and is similar to the behavior of $\left(\pi_{N} / \pi_{S}\right)^{\kappa}(t)$. The ratio decreases for $\kappa>1$, which means that less deleterious nonsynonymous mutations went to fixation - in accordance with observations about selection acting more efficiently in larger populations. However, $\omega^{\kappa}(t)$ is an accumulative measure over the time interval $[0, t]$, while $\left(\pi_{N} / \pi_{S}\right)^{\kappa}(t)$ reflects a snapshot of the strength 
of selection at time $t$. As a consequence, $\omega^{\kappa}(t)$ takes longer to reach its new equilibrium than $\left(\pi_{N} / \pi_{S}\right)^{\kappa}(t)$.

Another model to capture the impact of a change in population size on $\omega^{\kappa}(t)$ can be achieved by the weighted sum of the ancestral and the new equilibrium value (Eyre-Walker 2002). In our notation this reads $\omega_{\mathrm{w}}^{\kappa}(t):=$ $\left[t^{*} \omega^{1}+\left(t-t^{*}\right) \omega^{\kappa}\right] / t$ for equilibrium values $\omega^{1}$ and $\omega^{\kappa}$, respectively. This means, the approach based on the weighted sum does not explicitly model nonstationarity. To visualize the difference between $\omega^{\kappa}(t)$ and $\omega_{\mathrm{w}}^{\kappa}(t)$, the weighted fixation rate ratios are included as dashed lines in Fig. 2B. The nonequilibrium model derived in this study shows that $\omega^{\kappa}(t)$ takes longer to react to the change in population size and to reach the new equilibrium value compared to the approach of weighting the equilibrium values. This illustrates that ignoring the nonequilibrium phase leads to an underestimation of the time until the new equilibrium is reached.

Note that Fig. 2B shows the fixation rate ratio for a change in population size at time $t^{*}=1$. Changes at other time points can change the behavior severely. A change at $t^{*}=0$ for example, would lead to $\omega_{\mathrm{w}}^{\kappa}(t)=\omega^{\kappa}$ without reflecting any influence of the ancestral population. On the other hand, if the change in population size happens more recently in time, the contribution of the ancient population size becomes more pronounced (see Appendix Fig. E.6).

\section{Proxies of effective population size as measures of genetic drift}

In order to evaluate the efficacy of selection during nonequilibrium we relate the above-introduced measures of selection to estimates of the effective population size. Since there are various ways to define $N_{\text {eff }}$, it is funda- 
A

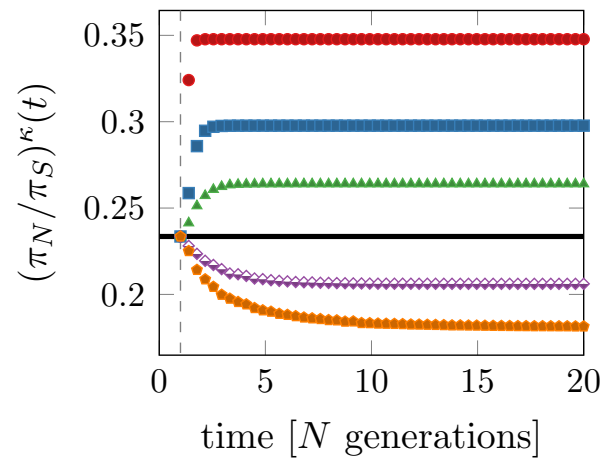

B

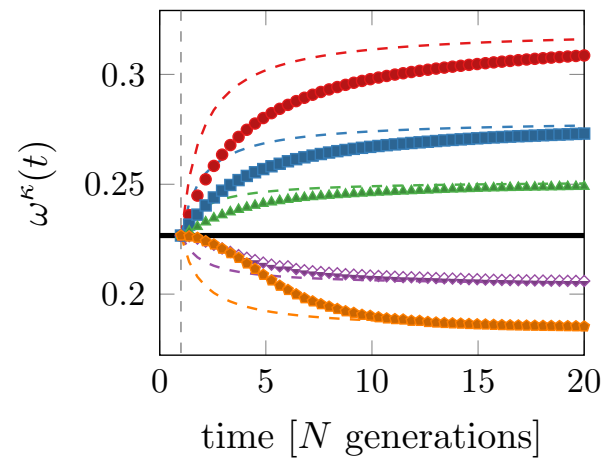

$\kappa=0.1 \quad \square \kappa=0.25 \quad \Delta \kappa=0.5 \quad-\kappa=1 \quad \diamond \kappa=2 \quad \Delta \kappa=4$

Figure 2: Measures of selection for different values of $\kappa$ as functions of time. Panel A: the ratio of nonsynonymous and synonymous diversity $\left(\pi_{N} / \pi_{S}\right)^{\kappa}(t)$. Panel B: the fixation rate ratio $\omega^{\kappa}(t)$. For comparison, colored, dashed curves represent the weighted fixation rate ratio $\omega_{\mathrm{w}}^{\kappa}(t)$. Vertical, dashed lines indicate the time $t^{*}=1$. Parameters: $\theta=1$, and $a=0.15$ and $a b=2500$ for the DFE.

mental to discuss the differences and to assess which of the definitions are relevant to relate to $\omega^{\kappa}(t)$ and $\left(\pi_{N} / \pi_{S}\right)^{\kappa}(t)$ in our modeling approach. The most commonly considered concepts of $N_{\text {eff }}$ among others are variance and inbreeding (Wright 1931, 1938; Crow 1954; Crow and Kimura 1970), coalescent (Lynch and Conery 2003), and eigenvalue effective population size (Ewens 1969, 1979, 1982). The properties, that these concepts aim to model, are the variance in allele frequencies over time due to random genetic drift, the average inbreeding coefficient, the rate of coalescence of neutral alleles, and the leading non-unit eigenvalue of the allele frequency transition matrix.

We here focus on the harmonic mean effective population size over $[0, t]$ (Wright 1938; Karlin 1968) and the pairwise synonymous nucleotide diversity (Lynch and Conery 2003; Wakeley and Sargsyan 2008; Ellegren and Galtier 
2016). The harmonic mean effective size over $[0, t]$ is a representative of variance effective population size and defined as the average of genetic drift over the time interval $[0, t]$ with $t^{*} \in[0, t]$,

$$
N_{\mathrm{eff}}^{h}(t):=\left(\frac{1}{t} \int_{0}^{t} \frac{1}{N_{\kappa}(s)} d s\right)^{-1}=\frac{\kappa N t}{t^{*}(\kappa-1)+t},
$$

with $N_{\text {eff }}^{h}\left(t^{*}\right)=N$, and $N_{\text {eff }}^{h}(t) \rightarrow \kappa N$ for $t$ large. This means the ancestral population size $N$ loses its influence on $N_{\text {eff }}^{h}(t)$ the further in the past the change took place. Overall the quantity is mainly controlled by the smaller population size (Wright 1938). If $\kappa$ is constant over $[0, t]$, then $N_{\text {eff }}^{h}(t)=\kappa N$. Also, in view of the genetic drift term in Eq. (1) - the variance term of the SDE - the parameter $\kappa$ at time $t$ multiplied by $N$ can be seen as variance effective population size at time $t$.

The scaled pairwise synonymous diversity, $N_{\mathrm{eff}}^{\pi}(t):=\pi_{S}^{\kappa}(t) /(2 \mu)$, where $\mu:=\theta / N$ is the mutation rate per generation and individual, is an estimate of effective population size based on nucleotide variation, often also perceived as coalescent effective population size (Lynch and Conery 2003; Wakeley and Sargsyan 2008). We note that the definition of coalescent effective population size is not consistent in literature (Sjödin et al. 2004). We here follow the definition of Lynch and Conery (2003); Wakeley and Sargsyan (2008).

With the two measures, $N_{\text {eff }}^{h}(t)$ and $N_{\text {eff }}^{\pi}(t)$, of effective population size at hand, we investigate and compare how a change in population size is reflected in each of them. We consider two scenarios, an ancient change at $t^{*}=1$ and a recent change at $t^{*}=18$. For this purpose, $N_{\text {eff }}^{h}(t)$ is plotted against $N_{\mathrm{eff}}^{\pi}(t)$ as parametric curve of time, $t \geq t^{*}$, for an ancient (Fig. 3A) and recent (Fig. 3B and Appendix Fig. E.7) change in population size. The nonequilibrium behavior is compared to the equilibrium values, i.e. 
$N_{\text {eff }}^{h}(t)=N_{\text {eff }}^{\pi}(t)=\kappa N$, as functions of $\kappa$. To explain Fig. 3 more explicitly, starting from a common initial equilibrium value at time $t^{*}$ indicated as black cross on the dashed equilibrium line, the relationship between $N_{\text {eff }}^{h}(t)$ and $N_{\text {eff }}^{\pi}(t)$ is shown for different values of $\kappa$ for $t^{*}<t<20$ as colored curves.

For an ancestral change, it seems that both proxies mirror the change in size to a large degree. However, $N_{\text {eff }}^{h}(t)$ reaches the new equilibrium value more slowly compared to $N_{\text {eff }}^{\pi}(t)$. This holds in particular for $\kappa>1$, leading to the presumption that the more a population increases, the slower the new equilibrium is reached and vice versa. For a recent change in population size, the difference between the two estimates is much more striking and we observe that there is hardly any correlation between them. The proxy $N_{\text {eff }}^{\pi}(t)$ reacts quickly to a change in population size, as expected for a measure relevant at the microevolutionary timescale. The harmonic mean effective population size, $N_{\text {eff }}^{h}(t)$, reacts more slowly and its variation is very narrow in comparison to $N_{\text {eff }}^{\pi}(t)$. This suggests that $N_{\text {eff }}^{\pi}(t)$ reflects a recent change in population size better than $N_{\mathrm{eff}}^{h}(t)$. Considering the analytical definitions of the two estimates, one can additionally state that changes which happened before time zero, i.e. $t^{*} \leq 0$, will not at all be captured by $N_{\text {eff }}^{h}(t)$ but potentially by $N_{\text {eff }}^{\pi}(t)$ given $t$ is small enough such that the AFS has not reached its new equilibrium.

\section{Relationship between effective population size and measures of nat- ural selection}

We investigate the selection-drift relationship after a change in population size and compare it to the equilibrium behavior. Therefore, we relate the two estimates $N_{\text {eff }}^{h}(t)$ and $N_{\text {eff }}^{\pi}(t)$ to the fixation rate ratio, $\omega^{\kappa}(t)$, and the 
A

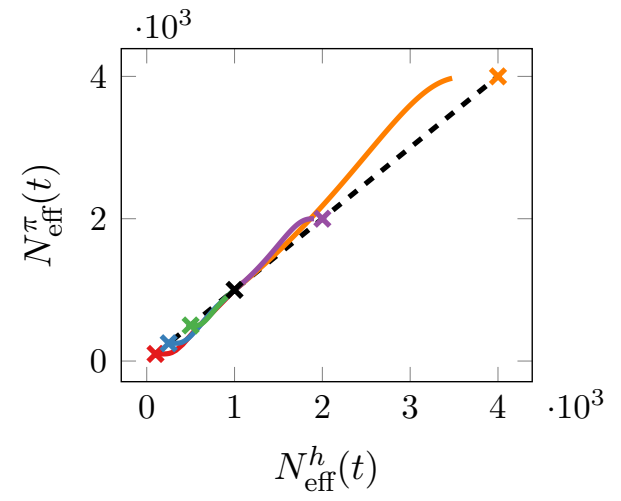

B

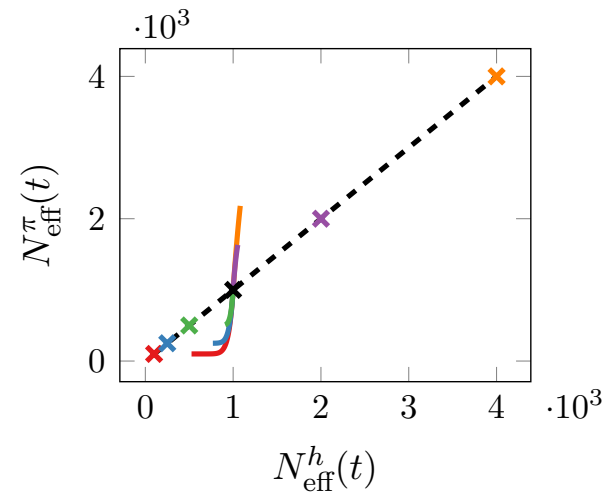

- - equilibrium $-\kappa=0.1-\kappa=0.25-\kappa=0.5-\kappa=2-\kappa=4$

Figure 3: The effective population size based on nucleotide variation, $N_{\mathrm{eff}}^{\pi}(t)$, versus the harmonic mean effective population size, $N_{\text {eff }}^{h}(t)$, as parametric curves of time, $t^{*} \leq t \leq 20$, for different values of $\kappa$. Panel A shows an ancient change in population size at $t^{*}=1$, panel $\mathrm{B}$ a recent change at $t^{*}=18$. Black, dashed lines show the expected behavior in equilibrium populations. Black crosses at $N_{\text {eff }}^{h}\left(t^{*}\right)=N_{\text {eff }}^{\pi}(t)=1000$ mark the time of change in population size, $t^{*}$. Colored crosses indicate the expected equilibrium value for each $\kappa$. Parameters $\theta=1$ and $N=1000$.

ratio of nucleotide diversity, $\left(\pi_{N} / \pi_{S}\right)^{\kappa}(t)$, as parametric curves of time after an ancient $\left(t^{*}=1\right.$, Fig. 4) and recent $\left(t^{*}=18\right.$, Fig. 5) change in size. To evaluate the discrepancy between the nonequilibrium behavior and the expected equilibrium balance between genetic drift and selection, we indicate $\omega^{\kappa}$ and $\left(\pi_{N} / \pi_{S}\right)^{\kappa}$ as functions of $\kappa$.

First, we consider an ancient change in population size (Fig. 4). This reveals a clear deviation from the equilibrium prediction of the nearly neutral theory for a relevant time period after the change in population size. For an increase in population size, $\omega^{\kappa}(t)$ is larger than expected in equilibrium, implying that more nonsynonymous and hence deleterious mutations get fixed during the nonequilibrium phase than during equilibrium, see 
Figs. 4A and 4B. This implies that the efficacy of selection is limited during the nonequilibrium period. The reverse is true for a decrease in size. Moreover, the discrepancy between nonequilibrium and equilibrium conditions is larger, the stronger the change in population size. In addition, the deviation from the selection-drift relationship appears more pronounced when comparing $\omega^{\kappa}(t)$ and $N_{\text {eff }}^{\pi}(t)$ than $\omega^{\kappa}(t)$ and $N_{\text {eff }}^{h}(t)$. For $\left(\pi_{N} / \pi_{S}\right)^{\kappa}(t)$ the deviation from equilibrium is less striking - even when relating to $N_{\text {eff }}^{h}(t)$ in Fig. $4 \mathrm{C}$. But still, there exists a deviation which notably is the other way around than for $\omega^{\kappa}(t)$. While for $\kappa<1$ the measure $\omega^{\kappa}(t)$ falls below the equilibrium expectation, the measure $\left(\pi_{N} / \pi_{S}\right)^{\kappa}(t)$ for a given $N_{\text {eff }}$ is larger than in equilibrium. The reverse holds for $\kappa>1$.

For a recent change in population size in Fig. 5, when using the fixation rate ratio $\omega^{\kappa}(t)$ as measure of selection, the selection-drift relationship becomes very weak (see Figs. 5A and 5B). The lack of a clear relationship between $\omega^{\kappa}(t)$ and $N_{\text {eff }}$ can be explained by the slow reaction of $\omega^{\kappa}(t)$ on the change in population size. Since also $N_{\text {eff }}^{h}(t)$ reacts slow on the change in population size, $N_{\mathrm{eff}}^{h}(t)$ and $\omega^{\kappa}(t)$ are both close to the ancestral value, and the lack of the selection-drift relationship is less apparent. However, it becomes clearly visible when using $N_{\text {eff }}^{\pi}(t)$ as estimate of genetic drift.

The measures $\left(\pi_{N} / \pi_{S}\right)^{\kappa}(t)$ and $N_{\text {eff }}^{\pi}(t)$ react much faster on a change in population size, which is especially eye catching in the comparison of Figs. 5A and 5D. Figure 5D shows that there is hardly any deviation from the expected equilibrium relationship since $\left(\pi_{N} / \pi_{S}\right)^{\kappa}(t)$ and $N_{\text {eff }}^{\pi}(t)$ both react quickly to a change in population size and are hence close to their equilibrium expectation. This means comparison of microevolutionary measures of natural 
A

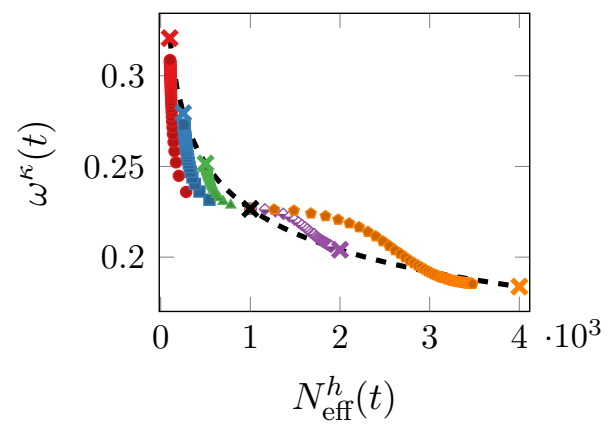

C

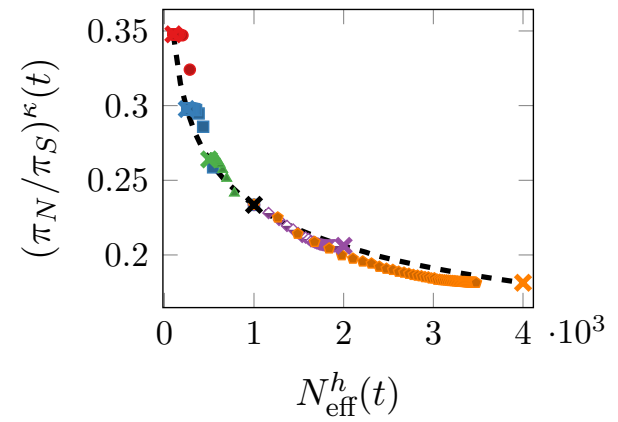

B

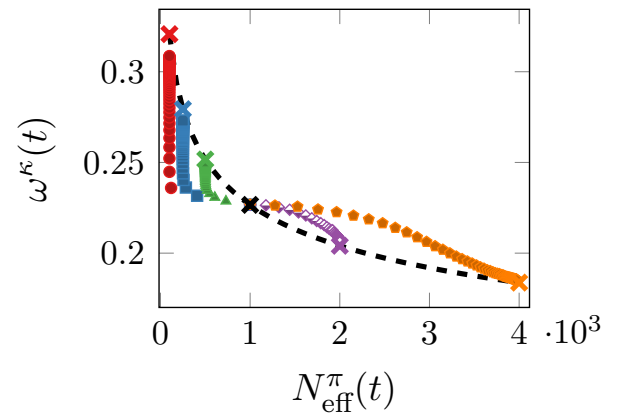

D

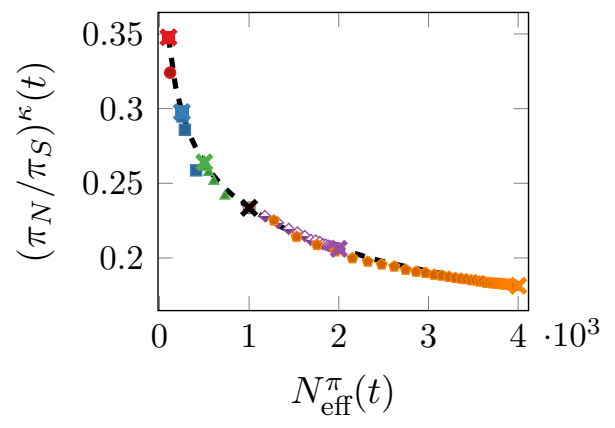

$\kappa=0.1 \quad \kappa=0.25 \Delta \kappa=0.5 \Delta \kappa=2 \Delta \kappa=4 \quad \cdots$ - $\quad \Delta$ equilibrium

Figure 4: Measures of selection versus proxies of $N_{\mathrm{eff}}$ as parametric curves of time, $1 \leq t \leq$ 20 , after an ancient change in population size at $t^{*}=1$ for different values of $\kappa$. Panels A and C: genetic drift estimated by the harmonic mean effective population size, $N_{\mathrm{eff}}^{h}(t)$. Panels B and D: genetic drift estimated by $N_{\text {eff }}^{\pi}(t)$. Black, dashed lines show the expected relation in equilibrium populations. Black crosses at $N_{\text {eff }}^{h}\left(t^{*}\right)=N_{\text {eff }}^{\pi}(t)=1000$ indicate the time of change in population size, $t^{*}$. Colored crosses indicate the expected equilibrium value for each $\kappa$. Parameters $a=0.15$ and $a b=2500$ in the DFE, $N=1000$, and $\theta=1$. 
A

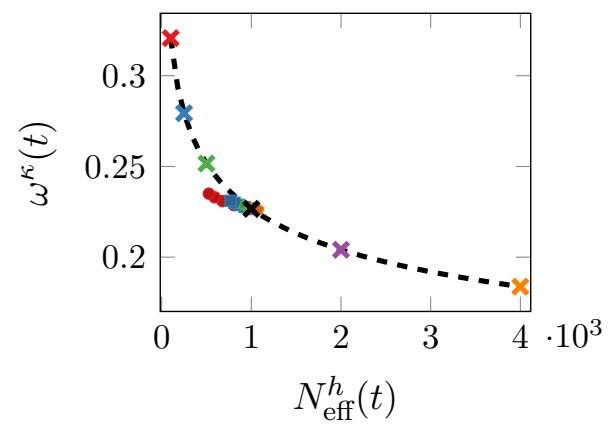

C

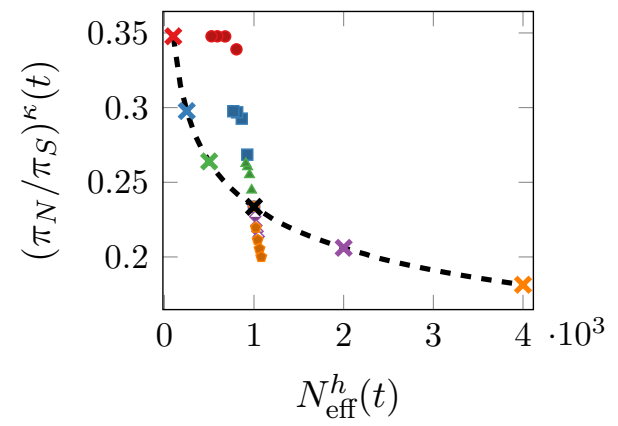

B

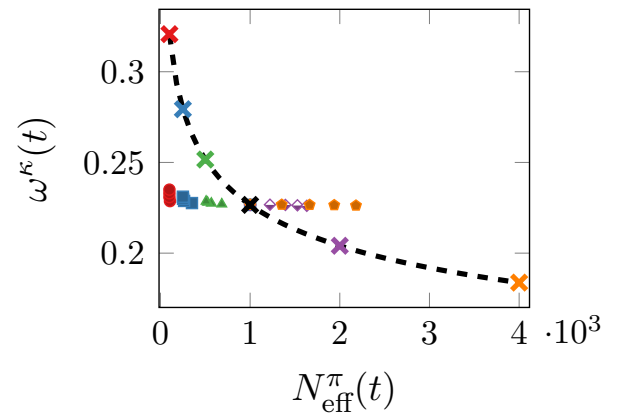

D

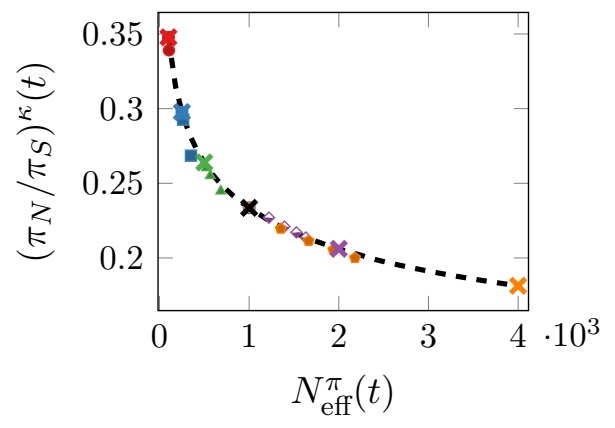

$\kappa=0.1 \quad \kappa=0.25 \Delta \kappa=0.5 \Delta \kappa=2 \Delta \kappa=4 \quad \cdots$ - $\quad \Delta$ equilibrium

Figure 5: Measures of selection versus proxies of $N_{\text {eff }}$ as parametric curves of time, $18 \leq$ $t \leq 20$, after a recent change in population size at $t^{*}=18$ for different values of $\kappa$. Panels A and C: genetic drift estimated by the harmonic mean effective population size, $N_{\mathrm{eff}}^{h}(t)$. Panels B and D: genetic drift estimated by $\pi_{S}^{\kappa}(t)$. Black, dashed lines show the expected relation in equilibrium populations. Black crosses at $N_{\text {eff }}^{h}\left(t^{*}\right)=N_{\text {eff }}^{\pi}(t)=1000$ indicate the time of change in population size, $t^{*}$. Colored crosses indicate the expected equilibrium value for each $\kappa$. Parameters $a=0.15$ and $a b=2500$ in the DFE, $N=1000$, and $\theta=1$. 
selection and genetic drift recover the equilibrium selection-drift relationship quickly. In contrast, comparison of macroevolutionary measures of natural selection and genetic drift (Fig. 5A) appear not applicable to capture recent changes in population size. If a microevolutionary measure is compared with a macroevolutionary measure after a recent change in population size, this can essentially lead to a lack of a selection-drift relationship (Fig. 5B), or stronger observed variation in natural selection than observed variation in genetic drift (Fig. 5C), dependent on the choice of combination.

Overall, we can recognize that the prediction of the nearly neutral theory, i.e. a negative correlation between measures of selection and population size, holds even after a change in population size. But the strength of relationship is clearly influenced dependent on what measures of selection and $N_{\text {eff }}$ are chosen. The analysis and discussion of the various approaches to $N_{\text {eff }}$ confirm that it is not trivial to choose an appropriate estimate that reflects the population behavior. Our results suggest that $N_{\text {eff }}^{\pi}(t)$ and $\left(\pi_{N} / \pi_{S}\right)^{\kappa}(t)$ as microevolutionary measures reflect a recent change in population size well but quickly loose the knowledge about it. In contrast, the macroevolutionary measures $N_{\text {eff }}^{h}(t)$ and $\omega^{\kappa}(t)$ need more time to react to the change but are longer influenced by the ancestral population size. We can further conclude that, in order to investigate the selection-drift relationship, it seems advisable to correlate microevolutionary measures of $N_{\text {eff }}$ with microevolutionary measures of selection in case of a recent change in population size. For an ancient change, microevolutionary measures of $N_{\text {eff }}$ should be correlated with microevolutionary measures of selection and macroevolutionary measures of $N_{\text {eff }}$ with macroevolutionary measures of selection. Moreover, it could be of 
interest to investigate and compare the selection-drift relationship of microand macroevolutionary measures. If the observed relationships show a different behavior, this could be indicative of a nonequilibrium condition.

Obviously, data availability is an important prerequisite for such an analysis. Microevolutionary measures of genetic drift and natural selection can be directly computed based on population re-sequencing data (Muyle et al. 2020). On the other hand, in practice $N_{\text {eff }}^{h}(t)$ as a macroevolutionary measure of $N_{\text {eff }}$ is difficult to assess based on genomic data. Instead, life-history traits, such as body mass or propagule size, are commonly used as proxies for long-term $N_{\text {eff }}$ (e.g. Waples 2016; Tabak et al. 2018; Kutschera et al. 2020). The lack of a distantly related reference species can further hinder the computation of macroevolutionary measures of natural selection (Mugal et al. 2020; Muyle et al. 2020). Such limitations are often unavoidable, but our results highlight that it is important to be aware of the measures used in different studies when making comparisons among studies. When comparing the relationship between the efficacy of selection and the effects of genetic drift among different studies, as for example asexually and sexually reproducing species, or domesticated versus wild species, it could therefore be advisable to take the nature of measures of effective population size and natural selection into account (Brandvain and Wright 2016).

\section{Conclusion}

Addressing our initial question of how a change in population size affects $\left(\pi_{N} / \pi_{S}\right)^{\kappa}(t)$ and $\omega^{\kappa}(t)$, both measures show a similar behavior but differ in how quickly they react to the change. For a population decrease, both ratios increase and vice versa, but $\left(\pi_{N} / \pi_{S}\right)^{\kappa}(t)$ as a microevolutionary measure re- 
acts much faster to the change than the macroevolutionary measure $\omega^{\kappa}(t)$. Second, we asked if the selection-drift relationship predicted by the nearly neutral theory also holds in a nonequilibrium. For this purpose, we compared the relationship between measures of genetic drift and selection after the change in population size with the expected equilibrium balance. Our analytical results reveal that the expected negative relationship between population size and measures of selection, such as $\left(\pi_{N} / \pi_{S}\right)^{\kappa}(t)$ and $\omega^{\kappa}(t)$, is still valid, but we observe a clear deviation from the expected equilibrium balance during nonequilibrium periods. Moreover, we conclude that since measures of micro- and macroevolution show different behaviors, it is important to be aware of this fact when making inference and comparing results among studies - different observations could arise from using measures at different evolutionary timescales.

We built our model of a nonequilibrium scenario on several simplifying assumptions of which one is the focus on a single change in population size. The advantage of such narrow focus is that interpretations can be done more easily and straightforward. On the other hand, the framework we presented here is only directly comparable to a limited number of empirical scenarios, such as (but not limited to) reductions in effective population size due to isolation of island and mainland populations (Woolfit and Bromham 2005; Wang et al. 2014; Leroy et al. 2020). In addition, it might also be relevant to study periodic changes in population size as for example done by Rousselle et al. (2018) using simulations. Our analytical modeling approach, especially the derivation of a nonequilibrium AFS in the Poisson random field framework, may be extended to such situations. Periodic changes in population 
size would entail a sequence $t_{k}^{*}=k T$, for $k=0,1, \ldots$, of time points of period $T$ and a sequence $\kappa_{0}, \kappa_{1}, \ldots$ of size parameters making up a new step function $N_{\kappa}$. For $t \in\left[t_{n-1}^{*}, t_{n}^{*}\right)$, the more general nonequilibrium AFS extending Eq. (10) is

$$
\begin{aligned}
& \lim _{N \rightarrow \infty} \mathbb{E}\left\langle\mathcal{X}_{t}^{N_{\kappa}}, f\right\rangle \\
& =\theta \int_{0}^{1} f(y) \omega_{\gamma, \kappa_{0}} \psi_{\gamma, \kappa_{0}}(y) d y+\theta f(1) \int_{0}^{t} \sum_{k=1}^{n} \omega_{\gamma, \kappa_{k-1}} 1_{\left\{t_{k-1}^{*}<s \leq t_{k}^{*}\right\}} d s \\
& +\theta \sum_{k=1}^{n} \int_{0}^{1}\left(f(y)-\mathbb{E}_{y}^{\gamma, \kappa_{k-1}} f\left(\xi_{t_{k}^{*} \wedge t}^{t_{k}^{*}}\right)\right)\left(\omega_{\gamma, \kappa_{k}} \psi_{\gamma, \kappa_{k}}(y)-\omega_{\gamma, \kappa_{k-1}} \psi_{\gamma, \kappa_{k-1}}(y)\right) d y .
\end{aligned}
$$

In fact, this AFS is not restricted to periodically changing environments, and hence may be used to develop the further case of allowing a prescribed, continuously varying, deterministic, scaled population size, such that $N(t) / N \rightarrow$ $\kappa(t)$.

Apart from change in population size, there are other mechanisms that can cause a demographic nonequilibrium and affect the selection-drift balance. Examples of such mechanisms are population structure or migration. That the latter scenario can impact inference from genomic data is exemplified by the inference of estimates of gene flow in nonequilibrium conditions (e.g. Austin et al. 2004; Pinho et al. 2008). It could therefore be interesting to extend the description of the nonequilibrium AFS in order to incorporate migration and investigate its effect on the selection-drift relationship. Another evolutionary force that could impact the selection-drift relationship is recombination (Hill and Robertson 1966; Hollister et al. 2014; Campos et al. 2014). Our analytical model relies on the assumption of free recombination between sites, i.e. no linkage between sites. We show in Mugal et al. (2020) 
that measures of natural selection are affected by recombination, but that evolutionary trends of measures of selection are robust to the assumption of free recombination at least in equilibrium. However, evolutionary changes in the recombination landscape cause a deviation from equilibrium and could be a relevant topic for the future.

Finally, we restricted our analysis to purifying selection based on the argument that positive selection can be neglected due to the rarity of advantageous mutants on a genome-wide scale. This argument is still under debate (Kern and Hahn 2018; Jensen et al. 2018), and several empirical studies argue for a more significant role of positive selection at the genome-wide scale, (e.g. Fay et al. 2002; Begun et al. 2007; Lefebure and Stanhope 2009; Bazykin and Kondrashov 2012; Williamson et al. 2014). Looking at the role of positive selection could in particular be of interest when investigating the behavior of specific genes that are candidates for episodic positive selection (Schmid and Tautz 1997; Zheng et al. 2004; Nevado et al. 2019), for example immune genes (Enard et al. 2016) and genes involved in reproduction (Singh and Kulathinal 2000; Swanson and Vacquier 2002), rather than looking at the genome-wide average. The flexibility of our model allows for an extension to model advantageous mutations by considering for example a mixed DFE (Mugal et al. 2020). However, change in population size might push the population closer or further away from its fitness optimum dependent on the direction of the change, which would induce a change in the DFE. Such model extension would require a different setting, such as a mutation-selection-drift framework (Halpern and Bruno 1998; Spielman and Wilke 2015; Jones et al. 2016), which is a non-trivial extension. 
To conclude, some of the model assumptions constitute simplifying assumptions that lead to a narrow focus. The crucial advantage of such a narrow focus is that exact analytical solutions can be derived and that valuable conceptual understanding can be gained based on straightforward interpretations. The framework we presented here is also flexible and allows for various modifications, which gives the opportunity to investigate different extensions in some future work.

ACKNOWLEDGMENTS

The authors thank Martin Lascoux for valuable discussions and David Widmann for generous advice on implementing simulations in the Julia programming language. C.F.M. is funded by grants to Hans Ellegren from the Swedish Research Council (2013/08271) and Knut and Alice Wallenberg Foundation.

Austin, J. D., S. C. Lougheed, and P. T. Boag, 2004 Controlling for the Effects of History and Nonequilibrium Conditions in Gene Flow Estimates in Northern Bullfrog (Rana catesbeiana) Populations. Genetics 168: 14911506.

Bazykin, G. A. and A. S. Kondrashov, 2012 Major role of positive selection in the evolution of conservative segments of Drosophila proteins. Proceedings of the Royal Society B: Biological Sciences 279: 3409-3417. 
Begun, D. J., A. K. Holloway, K. Stevens, L. W. Hillier, Y.-P. Poh, et al., 2007 Population Genomics: Whole-Genome Analysis of Polymorphism and Divergence in Drosophila simulans. PLoS Biology 5: e310.

Bolívar, P., L. Guéguen, L. Duret, H. Ellegren, and C. F. Mugal, 2019 GCbiased gene conversion conceals the prediction of the nearly neutral theory in avian genomes. Genome Biology 20: 1-13.

Brandvain, Y. and S. I. Wright, 2016 The Limits of Natural Selection in a Nonequilibrium World. Trends in Genetics 32: 201-210.

Campos, J. L., D. L. Halligan, P. R. Haddrill, and B. Charlesworth, 2014 The Relation between Recombination Rate and Patterns of Molecular Evolution and Variation in Drosophila melanogaster. Molecular Biology and Evolution 31: 1010-1028.

Chen, J., S. Glémin, and M. Lascoux, 2017 Genetic Diversity and the Efficacy of Purifying Selection across Plant and Animal Species. Molecular Biology and Evolution 34: 1417-1428.

Crow, J. F., 1954 Breeding structure of populations. II. Effective population number. Statistics and Mathematics in Biology, Iowa State College Press, Ames, Iowa pp. 543-556.

Crow, J. F. and M. Kimura, 1970 An Introduction to Population Genetics Theory. Harper and Row, New York.

Ellegren, H. and N. Galtier, 2016 Determinants of genetic diversity. Nature Reviews Genetics 17: 422-433.

Elyashiv, E., K. Bullaughey, S. Sattath, Y. Rinott, M. Przeworski, et al., 2010 Shifts in the intensity of purifying selection: An analysis of genomewide polymorphism data from two closely related yeast species. Genome 
Research 20: 1558-1573.

Enard, D., L. Cai, C. Gwennap, and D. A. Petrov, 2016 Viruses are a dominant driver of protein adaptation in mammals. eLife $\mathbf{5}$.

Evans, S. N., Y. Shvets, and M. Slatkin, 2007 Non-equilibrium theory of the allele frequency spectrum. Theoretical Population Biology 71: 109-119.

Ewens, W., 1982 On the concept of the effective population size. Theoretical Population Biology 21: 373-378.

Ewens, W. J., 1969 Population Genetics. Methuen, London.

Ewens, W. J., 1979 Mathematical Population Genetics. Springer, New York.

Eyre-Walker, A., 2002 Changing Effective Population Size and the McDonald-Kreitman Test. Genetics 162: 2017-2024.

Eyre-Walker, A., M. Woolfit, and T. Phelps, 2006 The Distribution of Fitness Effects of New Deleterious Amino Acid Mutations in Humans. Genetics 173: 891-900.

Fay, J. C., G. J. Wyckoff, and C.-I. Wu, 2002 Testing the neutral theory of molecular evolution with genomic data from Drosophila. Nature 415: 1024-1026.

Figuet, E., B. Nabholz, M. Bonneau, E. Mas Carrio, K. NadachowskaBrzyska, et al., 2016 Life History Traits, Protein Evolution, and the Nearly Neutral Theory in Amniotes. Molecular Biology and Evolution 33: 15171527.

Fisher, R. A., 1930 The genetical theory of natural selection. The Clarendon Press.

Galtier, N. and M. Rousselle, 2020 How Much Does $N_{e}$ Vary Among Species? Genetics 216: 559-572. 
Goldman, N. and Z. Yang, 1994 A Codon-based Model of Nucleotide Substitution for Protein-coding DNA Sequences. Molecular Biology and Evolution 11: $725-736$.

Gordo, I. and F. Dionisio, 2005 Nonequilibrium model for estimating parameters of deleterious mutations. Physical Review E 71: 031907.

Halpern, A. L. and W. J. Bruno, 1998 Evolutionary distances for proteincoding sequences: modeling site-specific residue frequencies. Molecular Biology and Evolution 15: 910-917.

Hill, W. G. and A. Robertson, 1966 The effect of linkage on limits to artificial selection. Genetical Research 8: 269-294.

Hollister, J. D., S. Greiner, W. Wang, J. Wang, Y. Zhang, et al., 2014 Recurrent Loss of Sex Is Associated with Accumulation of Deleterious Mutations in Oenothera. Molecular Biology and Evolution 32: 896-905.

Hughes, A. L., 2008 Near-Neutrality: the Leading Edge of the Neutral Theory of Molecular Evolution. Annals of the New York Academy of Sciences 1133: $162-179$.

Jensen, J. D., B. A. Payseur, W. Stephan, C. F. Aquadro, M. Lynch, et al., 2018 The importance of the Neutral Theory in 1968 and 50 years on: A response to Kern and Hahn 2018. Evolution 73: 111-114.

Jones, C. T., N. Youssef, E. Susko, and J. P. Bielawski, 2016 Shifting Balance on a Static Mutation-Selection Landscape: A Novel Scenario of Positive Selection. Molecular Biology and Evolution 34: 391-407.

Kaj, I. and C. F. Mugal, 2016 The non-equilibrium allele frequency spectrum in a Poisson random field framework. Theoretical Population Biology 111: $51-64$. 
Karlin, S., 1968 Rates of approach to homozygosity for finite stochastic models with variable population size. The American Naturalist 102: 443-455.

Karlsson, E. K., D. P. Kwiatkowski, and P. C. Sabeti, 2014 Natural selection and infectious disease in human populations. Nature Reviews Genetics 15: 379-393.

Kern, A. D. and M. W. Hahn, 2018 The Neutral Theory in Light of Natural Selection. Molecular Biology and Evolution 35: 1366-1371.

Kimura, M., 1962 On the probability of fixation of mutant genes in a population. Genetics 47: 713 .

Kimura, M., 1964 Diffusion models in population genetics. Journal of Applied Probability 1: 177-232.

Kutschera, V. E., J. W. Poelstra, F. Botero-Castro, N. Dussex, N. J. Gemmell, et al., 2020 Purifying selection in corvids is less efficient on islands. Molecular biology and evolution 37: 469-474.

Lachapelle, J., N. Colegrave, and G. Bell, 2017 The effect of selection history on extinction risk during severe environmental change. Journal of Evolutionary Biology 30: 1872-1883.

Lefebure, T. and M. J. Stanhope, 2009 Pervasive, genome-wide positive selection leading to functional divergence in the bacterial genus Campylobacter. Genome Research 19: 1224-1232.

Leroy, T., M. Rousselle, M.-K. Tilak, A. Caizergues, C. Scornavacca, et al., 2020 Endemic island songbirds as windows into evolution in small effective population sizes. bioRxiv .

Loewe, L. and B. Charlesworth, 2006 Inferring the distribution of mutational effects on fitness in Drosophila. Biology Letters 2: 426-430. 
Lu, J., T. Tang, H. Tang, J. Huang, S. Shi, et al., 2006 The accumulation of deleterious mutations in rice genomes: a hypothesis on the cost of domestication. Trends in Genetics 22: 126-131.

Lumley, A. J., Ł. Michalczyk, J. J. Kitson, L. G. Spurgin, C. A. Morrison, et al., 2015 Sexual selection protects against extinction. Nature 522: 470473.

Lynch, M., R. Bürger, D. Butcher, and W. Gabriel, 1993 The Mutational Meltdown in Asexual Populations. Journal of Heredity 84: 339-344.

Lynch, M., J. Conery, and R. Burger, 1995 Mutation accumulation and the extinction of small populations. The American Naturalist 146: 489-518.

Lynch, M. and J. S. Conery, 2003 The Origins of Genome Complexity. Science 302: 1401-1404.

Mugal, C. F., V. E. Kutschera, F. Botero-Castro, J. B. Wolf, and I. Kaj, 2020 Polymorphism Data Assist Estimation of the Nonsynonymous over Synonymous Fixation Rate Ratio $\omega$ for Closely Related Species. Molecular Biology and Evolution 37: 260-279.

Mugal, C. F., J. B. Wolf, and I. Kaj, 2014 Why Time Matters: Codon Evolution and the Temporal Dynamics of $\mathrm{d} N / \mathrm{d} S$. Molecular Biology and Evolution 31: 212-31.

Muse, S. V. and B. S. Gaut, 1994 A Likelihood Approach for Comparing Synonymous and Nonsynonymous Nucleotide Substitution Rates, with Application to the Chloroplast Genome. Molecular Biology and Evolution 11: $715-724$.

Muyle, A., H. Martin, N. Zemp, M. Mollion, S. Gallina, et al., 2020 Dioecy is associated with high genetic diversity and adaptation rates in the plant 
genus silene. Molecular Biology and Evolution .

Nei, M. and W.-H. Li, 1979 Mathematical model for studying genetic variation in terms of restriction endonucleases. Proceedings of the National Academy of Sciences 76: 5269-5273.

Nevado, B., E. L. Wong, O. G. Osborne, and D. A. Filatov, 2019 Adaptive Evolution Is Common in Rapid Evolutionary Radiations. Current Biology 29: 3081-3086.e5.

Ohta, T., 1973 Slightly Deleterious Mutant Substitutions in Evolution. Nature 246: 96-98.

Ohta, T., 1976 Role of Very Slightly Deleterious Mutations in Molecular Evolution and Polymorphism. Theoretical Population Biology 10: 254275 .

Ohta, T., 1992 The nearly neutral theory of molecular evolution. Annual Review of Ecology and Systematics 23: 263-286.

Pinho, C., D. J. Harris, and N. Ferrand, 2008 Non-equilibrium estimates of gene flow inferred from nuclear genealogies suggest that Iberian and North African wall lizards (Podarcis spp.) are an assemblage of incipient species. BMC Evolutionary Biology 8.

Renaut, S. and L. H. Rieseberg, 2015 The Accumulation of Deleterious Mutations as a Consequence of Domestication and Improvement in Sunflowers and Other Compositae Crops. Molecular Biology and Evolution 32: 22732283.

Rousselle, M., M. Mollion, B. Nabholz, T. Bataillon, and N. Galtier, 2018 Overestimation of the adaptive substitution rate in fluctuating populations. Biology letters 14: 20180055. 
Sawyer, S. A. and D. L. Hartl, 1992 Population Genetics of Polymorphism and Divergence. Genetics 132: 1161-1176.

Schmid, K. J. and D. Tautz, 1997 A screen for fast evolving genes from Drosophila. Proceedings of the National Academy of Sciences 94: 97469750 .

Seppälä, O., 2015 Natural selection on quantitative immune defence traits: a comparison between theory and data. Journal of Evolutionary Biology 28: $1-9$.

Singh, R. S. and R. J. Kulathinal, 2000 Sex gene pool evolution and speciation: A new paradigm. Genes \& Genetic Systems 75: 119-130.

Sjödin, P., I. Kaj, S. Krone, M. Lascoux, and M. Nordborg, 2004 On the Meaning and Existence of an Effective Population Size. Genetics 169: 1061-1070.

Spielman, S. J. and C. O. Wilke, 2015 The Relationship between $d N / d S$ and Scaled Selection Coefficients. Molecular Biology and Evolution 32: 10971108.

Swanson, W. J. and V. D. Vacquier, 2002 The rapid evolution of reproductive proteins. Nature Reviews Genetics 3: 137-144.

Tabak, M. A., C. T. Webb, and R. S. Miller, 2018 Propagule size and structure, life history, and environmental conditions affect establishment success of an invasive species. Scientific Reports $\mathbf{8}$.

Wakeley, J. and O. Sargsyan, 2008 Extensions of the Coalescent Effective Population Size. Genetics 181: 341-345.

Wang, J., E. Santiago, and A. Caballero, 2016 Prediction and estimation of effective population size. Heredity 117: 193-206. 
Wang, S., W. Zhu, X. Gao, X. Li, S. Yan, et al., 2014 Population size and time since island isolation determine genetic diversity loss in insular frog populations. Molecular Ecology 23: 637-648.

Waples, R. S., 2005 Genetic estimates of contemporary effective population size: to what time periods do the estimates apply? Molecular Evolution 14: $3335-3352$.

Waples, R. S., 2016 Life-history traits and effective population size in species with overlapping generations revisited: the importance of adult mortality. Heredity 117: 241-250.

Williamson, R. J., E. B. Josephs, A. E. Platts, K. M. Hazzouri, A. Haudry, et al., 2014 Evidence for Widespread Positive and Negative Selection in Coding and Conserved Noncoding Regions of Capsella grandiflora. PLoS Genetics 10: e1004622.

Wolf, J. B., A. Künstner, K. Nam, M. Jakobsson, and H. Ellegren, 2009 Nonlinear Dynamics of Nonsynonymous $\left(d_{N}\right)$ and Synonymous $\left(d_{S}\right)$ Substitution Rates Affects Inference of Selection. Genome Biology and Evolution 1: $308-319$.

Woolfit, M. and L. Bromham, 2005 Population size and molecular evolution on islands. Proceedings of the Royal Society B: Biological Sciences 272: $2277-2282$.

Wright, S., 1931 Evolution in mendelian populations. Genetics 16: 97-159.

Wright, S., 1938 Size of population and breeding structure in relation to evolution. Science 87: 430-431.

Zheng, Y., R. J. Roberts, and S. Kasif, 2004 Identification of genes with fast-evolving regions in microbial genomes. Nucleic Acids Research 32: 
$835 \quad 6347-6357$.

836 Živković, D., M. Steinrücken, Y. S. Song, and W. Stephan, 2015 Transi-

${ }_{837}$ tion Densities and Sample Frequency Spectra of Diffusion Processes with

838 Selection and Variable Population Size. Genetics 200: 601-617.

839

Živković, D. and W. Stephan, 2011 Analytical results on the neutral non-

840 equilibrium allele frequency spectrum based on diffusion theory. Theoret-

$841 \quad$ ical Population Biology 79: 184-191. 


\section{A. THE AFS FOR THE EQUILIBRIUM CASE}

We begin with the reference case $\kappa=1$ of fixed population size and consider the Wright-Fisher diffusion $\xi^{s}=\left(\xi_{t}^{s}\right)_{t \geq s}, \xi_{s}^{s}=y$, with law $\mathbb{P}_{y}^{\gamma}\left(d \xi^{s}\right)$. To emphasize the initial value we may write $\left(\xi_{t}^{s, y}\right)_{t \geq s}$. The associated expectation operator $T_{t-s}^{\gamma} f(y)=\mathbb{E}_{y}^{\gamma}\left[f\left(\xi_{t}^{s}\right)\right], t \geq s$, satisfies the semigroup property $T_{s}^{\gamma}\left(T_{t-s}^{\gamma} f\right)(y)=T_{t} f(y)$, and the diffusion infinitesimal generator is the differential operator

$$
Q_{\gamma} f(x)=\gamma x(1-x) f^{\prime}(x)+\frac{1}{2} x(1-x) f^{\prime \prime}(x), \quad f \in \mathcal{D},
$$

for a suitable domain $\mathcal{D}$ of twice differentiable functions on the unit interval. We denote by $\mathcal{F}$ the class of real-valued bounded functions on $[0,1]$ with $f(0)=0$, satisfying $\int y^{-1}|f(y)| d y<\infty$, and put

$$
\widetilde{\mathcal{F}}=\left\{f \in \mathcal{F} \cap \mathcal{D}: Q_{\gamma} f \in \mathcal{F}\right\} .
$$

Similarly, $\mathcal{F}_{0}$ and $\widetilde{\mathcal{F}}_{0}$ denote the further restricted classes of functions which, in addition, satisfy $f(1)=0$. It is convenient to derive the following property first for the restricted class of functions vanishing at both boundary points, case i), and then make the necessary observations for handling the larger class of functions, as case ii).

Lemma 1. For each $t \geq 0$,

i) if $f \in \widetilde{\mathcal{F}}_{0}$ then $T_{t}^{\gamma} f \in \mathcal{F}_{0}$; and

ii) if $f \in \widetilde{\mathcal{F}}$ then $T_{t}^{\gamma} f \in \mathcal{F}$.

Proof. For statement i) let $f \in \widetilde{\mathcal{F}}_{0}$. Clearly, $T_{t}^{\gamma} f$ is bounded with $T_{t}^{\gamma} f(0)=$ $T_{t}^{\gamma} f(1)=0$. We need to show $\int_{0}^{1} x^{-1}\left|T_{t}^{\gamma} f\right|(x) d x<\infty$. 
862

863

$$
x^{-1} G_{\gamma}(x, y) y=\mathbb{1}_{\{x<y\}} x^{-1} q_{\gamma}(x) \psi_{\gamma}(y) y+\mathbb{1}_{\{x>y\}} x^{-1}\left(1-q_{\gamma}(x)\right) \tilde{\psi}_{\gamma}(y) y
$$

$$
T_{t}^{\gamma} f(x)=f(x)+\mathbb{E}_{x}^{\gamma}\left[\int_{0}^{t} Q_{\gamma} f\left(\xi_{r}\right) d r\right]
$$

and so

$$
\left|T_{t}^{\gamma} f(x)-f(x)\right| \leq \mathbb{E}_{x}^{\gamma}\left[\int_{0}^{t}\left|Q_{\gamma} f\left(\xi_{r}\right)\right| d r\right] .
$$

${ }_{864}$ Let $\tau$ be the absorption time of $\left(\xi_{t}\right)$. Then, since $Q_{\gamma} f \in \mathcal{F}_{0}$ by assumption,

$$
\mathbb{E}_{x}^{\gamma}\left[\int_{0}^{t}\left|Q_{\gamma} f\left(\xi_{r}\right)\right| d r\right]=\mathbb{E}_{x}^{\gamma}\left[\int_{0}^{t \wedge \tau}\left|Q_{\gamma} f\left(\xi_{r}\right)\right| d r\right] \leq \mathbb{E}_{x}^{\gamma}\left[\int_{0}^{\tau}\left|Q_{\gamma} f\left(\xi_{r}\right)\right| d r\right]
$$

so that

$$
\left|T_{t}^{\gamma} f(x)-f(x)\right| \leq \mathbb{E}_{x}^{\gamma}\left[\int_{0}^{\tau}\left|Q_{\gamma} f\left(\xi_{r}\right)\right| d r\right]=\int_{0}^{1}\left|Q_{\gamma} f(y)\right| G_{\gamma}(x, y) d y,
$$

where $G_{\gamma}(x, y)$ is the Green function of the Wright-Fisher diffusion. Hence,

$$
\begin{aligned}
\int_{0}^{1} x^{-1}\left|T_{t}^{\gamma} f(x)-f(x)\right| d x & \leq \int_{0}^{1} \int_{0}^{1}\left|Q_{\gamma} f(y)\right| x^{-1} G_{\gamma}(x, y) d y d x \\
& \leq \sup _{x, y}\left|x^{-1} G_{\gamma}(x, y) y\right| \int_{0}^{1} y^{-1}\left|Q_{\gamma} f(y)\right| d y
\end{aligned}
$$

and therefore, uniformly in $t$,

$$
\begin{aligned}
\int_{0}^{1} x^{-1}\left|T_{t}^{\gamma} f(x)\right| d x \leq & \int_{0}^{1} x^{-1}|f(x)| d x \\
& +\sup _{x, y}\left|x^{-1} G_{\gamma}(x, y) y\right| \int_{0}^{1} y^{-1}\left|Q_{\gamma} f(y)\right| d y .
\end{aligned}
$$

As the two integrals on the right hand side are finite by assumption, it remains to show that 
870 871 872

is bounded on the unit square $0 \leq x, y \leq 1$. Here, $q_{\gamma}(x)$ is defined in Eq. (2), $\psi_{\gamma}(y)$ in Eq. (4), and

$$
\tilde{\psi}_{\gamma}(y)=\frac{e^{2 \gamma y}-1}{\gamma y(1-y)}, \quad \gamma \neq 0, \quad \tilde{\psi}_{0}(y)=\frac{2}{1-y} .
$$

For $\gamma=0$,

$$
x^{-1} G_{0}(x, y) y=\mathbb{1}_{\{x<y\}} 2+\mathbb{1}_{\{x>y\}} \frac{1-x}{x} \frac{2 y}{1-y} \leq 2 .
$$

For $\gamma \neq 0$, the nonnegative function $x^{-1} G_{\gamma}(x, y) y$ has the representation and upper bound

$$
\begin{aligned}
& \frac{4 \gamma}{1-e^{-2 \gamma}}\left\{\mathbb{1}_{\{x<y\}} \frac{1-e^{-2 \gamma x}}{2 \gamma x} \frac{1-e^{-2 \gamma(1-y)}}{2 \gamma(1-y)}+\mathbb{1}_{\{x>y\}} \frac{e^{-2 \gamma x}-e^{-2 \gamma}}{2 \gamma x} \frac{e^{2 \gamma y}-1}{2 \gamma(1-y)}\right\} \\
& \leq \frac{4 \gamma}{1-e^{-2 \gamma}}\left\{\mathbb{1}_{\{x<y\}} \frac{1-e^{-2 \gamma x}}{2 \gamma x} \frac{1-e^{-2 \gamma(1-y)}}{2 \gamma(1-y)}+\mathbb{1}_{\{x>y\}} \frac{e^{-2 \gamma x}-e^{-2 \gamma}}{2 \gamma(1-x)} \frac{e^{2 \gamma y}-1}{2 \gamma y}\right\} .
\end{aligned}
$$

From here using straightforward estimates one obtains, for example,

$$
\sup _{0 \leq x, y \leq 1} x^{-1} G_{\gamma}(x, y) y \leq \frac{e^{2|\gamma|}-1}{|\gamma|},
$$

which concludes the proof that $T_{t}^{\gamma} f$ belongs to $\mathcal{F}_{0}$.

Turning to statement ii), by using Kaj and Mugal (2016, Lemma 1, Eq. (30)), we now have

$$
T_{t}^{\gamma} f(x)=f(x)+\mathbb{E}_{x}^{\gamma}\left[\int_{0}^{t \wedge \tau}\left(Q_{\gamma} f\left(\xi_{r}\right)-Q_{\gamma} f(1) q_{\gamma}\left(\xi_{r}\right)\right) d r\right]+Q_{\gamma} f(1) q_{\gamma}(x) t .
$$

Since $q_{\gamma}(1)=1$, the integrand function $y \mapsto Q_{\gamma} f(y)-Q_{\gamma} f(1) q_{\gamma}(y)$ belongs to $\mathcal{F}_{0}$. Hence we can proceed as above and obtain as replacement of Eq. (A.1),

$$
\begin{aligned}
\int_{0}^{1} x^{-1}\left|T_{t}^{\gamma} f(x)\right| d x & \leq \int_{0}^{1} x^{-1}|f(x)| d x+Q_{\gamma} f(1) t \int_{0}^{1} x^{-1} q_{\gamma}(x) d x \\
& +\sup _{x, y}\left|x^{-1} G_{\gamma}(x, y) y\right| \int_{0}^{1} y^{-1}\left|Q_{\gamma} f(y)-Q_{\gamma} f(1) q_{\gamma}(y)\right| d y,
\end{aligned}
$$


where the supremum is the same factor already treated under i). The observation

$$
\int_{0}^{1} x^{-1} q_{\gamma}(x) d x<\infty
$$

completes the proof.

The stationary AFS arises in the Poisson random field approach as a limiting intensity measure of a random Poisson measure which adds up the allele contributions from all mutations in the infinite past. Formally, let $\mathcal{N}_{N}\left(d s, d y, d \xi^{s}\right)$ be a Poisson measure on $\mathbb{R} \times[0,1] \times \mathcal{D}$ with intensity $N d s \delta_{1 / N}(d y) \mathbb{P}_{y}^{\gamma}\left(d \xi^{s}\right)$, and let $\mathcal{N}_{\gamma}(d y)$ be a Poisson random measure on $[0,1]$ with intensity measure $\omega_{\gamma} \psi_{\gamma}(y) d y$. Note that for simplicity we assume $\theta=1$ throughout Appendix A and Appendix B. For $f \in \mathcal{F}_{0}$, we have for fixed $t$ as $N \rightarrow \infty$ the convergence in distribution

$$
\int_{-\infty}^{t} \iint_{\mathcal{D}} f\left(\xi_{t}^{s, y}\right) \mathcal{N}_{N}\left(d s, d y, d \xi^{s, y}\right) \Rightarrow \int_{0}^{1} f(y) \mathcal{N}_{\gamma}(d y)
$$

and the associated convergence of expected values

$$
\mathbb{E}\left[\int_{-\infty}^{t} \iint_{\mathcal{D}} f\left(\xi_{t}^{s, y}\right) \mathcal{N}_{N}\left(d s, d y, d \xi^{s, y}\right)\right] \rightarrow \omega_{\gamma} \int_{0}^{1} f(y) \psi_{\gamma}(y) d y
$$

(Kaj and Mugal 2016, Section 2.4.). Each Poisson point in $\mathcal{N}_{N}$ represents the allele frequency of a mutation starting at time $s$ at a fraction $1 / N$ of the population. Taking all mutations with $s \leq t$ together and considering the resulting spectrum of polymorphic sites at $t$, we obtain $\mathcal{N}_{\gamma}$.

Next, we decompose the stationary Poisson measure $\mathcal{N}_{\gamma}$ at a fixed time $t \geq 0$, in two independent contributions by splitting up the mutation times, separating $s \leq 0$ and $0<s \leq t$, so that

$$
\mathbb{E}\left[\left\{\int_{-\infty}^{0}+\int_{0}^{t}\right\} \iint_{\mathcal{D}} f\left(\xi_{t}^{s, y}\right) \mathcal{N}_{N}\left(d s, d y, d \xi^{s, y}\right)\right] \rightarrow \omega_{\gamma} \int_{0}^{1} f(y) \psi_{\gamma}(y) d y .
$$


The limit of the first term in the decomposition is obtained in the following lemma, relation ii). The limit of the second term follows immediately, relation iii). Let $\mathcal{M}_{\gamma}\left(d y, d \xi^{0, y}\right)$ be a Poisson random measure on $[0,1] \times \mathcal{D}$ with intensity measure $m_{\gamma}\left(d y, d \xi^{0, y}\right)=\omega_{\gamma} \psi_{\gamma}(y) d y \mathbb{P}_{y}^{\gamma}\left(d \xi^{0}\right)$. The Poisson points $\left(y, \xi^{0, y}\right)$ are paths with initial state $\xi_{0}=y$ according to the stationary intensity $\omega_{\gamma} \psi_{\gamma}(y) d y$ and evolve as Wright-Fisher diffusions $\left(\xi_{t}^{0, y}\right)_{t \geq 0}$.

Lemma 2. For $f \in \widetilde{\mathcal{F}}_{0}$ we have as $N \rightarrow \infty$ the convergence in distribution, i) $\int_{-\infty}^{0} \iint_{\mathcal{D}} f\left(\xi_{t}^{s, y}\right) \mathcal{N}_{N}\left(d s, d y, d \xi^{s, y}\right) \Rightarrow \int_{[0,1] \times \mathcal{D}} f\left(\xi_{t}^{0, y}\right) \mathcal{M}_{\gamma}\left(d y, d \xi^{0, y}\right)$ and convergence of expected values,

ii) $\mathbb{E}\left[\int_{-\infty}^{0} \iint_{\mathcal{D}} f\left(\xi_{t}^{s, y}\right) \mathcal{N}_{N}\left(d s, d y, d \xi^{s, y}\right)\right] \rightarrow \int_{0}^{1} T_{t}^{\gamma} f(y) \omega_{\gamma} \psi_{\gamma}(y) d y$

and

iii) $\mathbb{E}\left[\int_{0}^{t} \iint_{\mathcal{D}} f\left(\xi_{t}^{s, y}\right) \mathcal{N}_{N}\left(d s, d y, d \xi^{s, y}\right)\right] \rightarrow \int_{0}^{1}\left(f(y)-T_{t}^{\gamma} f(y)\right) \omega_{\gamma} \psi_{\gamma}(y) d y$.

Proof. The logarithmic moment generating functional of the $N$-dependent Poisson integral equals

$\ln \mathbb{E}\left[\exp \left\{\int_{-\infty}^{0} \iint_{\mathcal{D}} f\left(\xi_{t}^{s, y}\right) \mathcal{N}_{N}\left(d s, d y, d \xi^{s, y}\right)\right\}\right]=N \int_{-\infty}^{0} \mathbb{E}_{1 / N}^{\gamma}\left[e^{f\left(\xi_{t}^{s}\right)}-1\right] d s$.

Here, conditioning on $\left\{\xi_{0}^{s}\right\}_{s<0}$,

$$
\begin{aligned}
& \int_{-\infty}^{0} \mathbb{E}_{x}^{\gamma}\left[e^{f\left(\xi_{t}^{s}\right)}-1\right] d s=\int_{-\infty}^{0} \mathbb{E}_{x}^{\gamma} \mathbb{E}_{\xi_{0}^{s}}^{\gamma}\left[e^{f\left(\xi_{t}^{0}\right)}-1\right] d s \\
& =\mathbb{E}_{x}^{\gamma}\left[\int_{-\infty}^{0} T_{t}\left(e^{f}-1\right)\left(\xi_{0}^{s}\right) d s\right]=\mathbb{E}_{x}^{\gamma}\left[\int_{0}^{\infty} T_{t}\left(e^{f}-1\right)\left(\xi_{r}\right) d r\right] .
\end{aligned}
$$

910 Since $f \in \widetilde{\mathcal{F}}_{0}$ we have $e^{f}-1 \in \widetilde{\mathcal{F}}_{0}$. By Lemma 1 i $), T_{t}\left(e^{f}-1\right) \in \mathcal{F}_{0}, t \geq 0$. 
911

912

913

Thus, the rightmost expression in Eq. (A.3) equals

$$
N \mathbb{E}_{1 / N}^{\gamma}\left[\int_{0}^{\tau} T_{t}^{\gamma}\left(e^{f}-1\right)\left(\xi_{r}\right) d r\right]=N \int_{0}^{1} G_{\gamma}(1 / N, y) T_{t}^{\gamma}\left(e^{f}-1\right)(y) d y .
$$

Therefore, as $N \rightarrow \infty$,

$$
\begin{array}{r}
N \int_{-\infty}^{0} \mathbb{E}_{1 / N}^{\gamma}\left[e^{f\left(\xi_{t}^{s}\right)}-1\right] d s \rightarrow \int_{0}^{1} T_{t}^{\gamma}\left(e^{f}-1\right)(y) \omega_{\gamma} \psi_{\gamma}(y) d y \\
=\ln \mathbb{E}\left[\exp \left\{\int_{[0,1] \times \mathcal{D}} f\left(\xi_{t}^{0, y}\right) \mathcal{M}_{\gamma}\left(d y, d \xi^{0, y}\right)\right\}\right]
\end{array}
$$

which shows convergence of the marginal distributions, for a fixed $t$. The convergence of the finite-dimensional distributions can be established as in Kaj and Mugal (2016, Proposition 2). Statement ii), which is

$$
N \int_{-\infty}^{0} \mathbb{E}_{1 / N}^{\gamma}\left[f\left(\xi_{t}^{s}\right)\right] d s \rightarrow \int_{0}^{1} T_{t}^{\gamma} f(y) \omega_{\gamma} \psi_{\gamma}(y) d y
$$

follows as above, now using that $T_{t}^{\gamma} f \in \mathcal{F}_{0}$. Then we obtain iii) by subtracting ii) from Eq. (A.2).

In view of Lemma $2 \mathrm{i}$ ), from now on we consider the allele frequency model given by the Poisson integral $\left\langle\mathcal{X}_{t}^{N}, f\right\rangle, t \geq 0$, defined by

$$
\left\langle\mathcal{X}_{t}^{N}, f\right\rangle=\int_{[0,1] \times \mathcal{D}} f\left(\xi_{t}^{0, y}\right) \mathcal{M}_{\gamma}\left(d y, d \xi^{0, y}\right)+\int_{0}^{t} \iint_{\mathcal{D}} f\left(\xi_{t}^{s, y}\right) \mathcal{N}_{N}\left(d s, d y, d \xi^{s, y}\right)
$$

Here, the site frequency paths enter the system either at $t=0$ according to a Poisson measure with intensity $\omega_{\gamma} \psi_{\gamma}(y) d y, 0 \leq y \leq 1$, or at constant rate $N d s, s \geq 0$, with initial frequency $1 / N$. In particular,

$$
\left\langle\mathcal{X}_{0}^{N}, f\right\rangle=\int_{0}^{1} f(y) \mathcal{N}_{\gamma}(d y)
$$

is independent of $N$ and we write $\mathcal{X}_{0}$. This setting allows us to analyze the effect of allele fixations, by dropping the restriction $f(1)=0$ required for 
$\widetilde{\mathcal{F}}_{0}$. The next result shows that fixations build up at an asymptotically linear rate over time with the deviation from linearity controlled by

$$
T_{t}^{\gamma} f(x)=\mathbb{P}_{x}^{\gamma}\left(\tau_{1} \leq t\right) f(1)+\mathbb{E}_{x}^{\gamma}\left[f\left(\xi_{t}\right), \tau>t\right] \rightarrow \frac{1-e^{-2 \gamma x}}{1-e^{-2 \gamma}} f(1), \quad t \rightarrow \infty
$$

where $\tau_{1}$ is fixation time and $\tau$ absorption time.

Lemma 3. For each $f \in \widetilde{\mathcal{F}}$ as $N \rightarrow \infty$
i) $\quad \mathbb{E}\left[\int_{0}^{t} \iint_{\mathcal{D}} f\left(\xi_{t}^{s, y}\right) \mathcal{N}_{N}\left(d s, d y, d \xi^{s, y}\right)\right]$
$\rightarrow \int_{0}^{1}\left(f(y)-T_{t}^{\gamma} f(y)\right) \omega_{\gamma} \psi_{\gamma}(y) d y+f(1) \omega_{\gamma} t$
ii) $\mathbb{E}_{\mathcal{X}_{0}}\left\langle\mathcal{X}_{t}^{N}, f\right\rangle \rightarrow\left\langle\mathcal{X}_{0}, T_{t}^{\gamma} f\right\rangle+\int_{0}^{1}\left(f(y)-T_{t}^{\gamma} f(y)\right) \omega_{\gamma} \psi_{\gamma}(y) d y+f(1) \omega_{\gamma} t$,
iii) $\mathbb{E}\left\langle\mathcal{X}_{t}^{N}, f\right\rangle \rightarrow \int_{0}^{1} f(y) \omega_{\gamma} \psi_{\gamma}(y) d y+f(1) \omega_{\gamma} t$.

Proof. We begin with the basic representation of the expected value given by

$$
\begin{aligned}
\mathbb{E} & {\left[\int_{0}^{t} \iint_{\mathcal{D}} f\left(\xi_{t}^{s, y}\right) \mathcal{N}_{N}\left(d s, d y, d \xi^{s, y}\right)\right] } \\
& =N \mathbb{E}_{1 / N}^{\gamma}\left[\int_{0}^{t} f\left(\xi_{t}^{s}\right) d s\right]=N \mathbb{E}_{1 / N}^{\gamma}\left[\int_{0}^{t} f\left(\xi_{u}^{0}\right) d u\right]
\end{aligned}
$$

1 and apply the rewriting

$$
\begin{aligned}
N \mathbb{E}_{1 / N}^{\gamma}\left[\int_{0}^{t} f\left(\xi_{u}\right) d u\right]= & N \mathbb{E}_{1 / N}^{\gamma}\left[\int_{0}^{t \wedge \tau}\left(f\left(\xi_{u}\right)-f(1) q_{\gamma}\left(\xi_{u}\right)\right) d u\right] \\
& +f(1) N q_{\gamma}(1 / N) t
\end{aligned}
$$

see Kaj and Mugal (2016, Section 6.3, Eq. (30)). Here, $N q_{\gamma}(1 / N) \rightarrow \omega_{\gamma}$, $N \rightarrow \infty$. Moreover, the function $g$ defined by $g(y)=f(y)-f(1) q_{\gamma}(y)$ 
934

935

941

belongs to $\widetilde{\mathcal{F}}_{0}$ and hence, by iii) in Lemma 2 ,

$$
\begin{aligned}
N \mathbb{E}_{1 / N}^{\gamma}\left[\int_{0}^{t \wedge \tau} g\left(\xi_{u}\right) d u\right] & =N \mathbb{E}_{1 / N}^{\gamma}\left[\int_{0}^{t} g\left(\xi_{u}\right) d u\right] \\
& \rightarrow \int_{0}^{1}\left(g(y)-T_{t}^{\gamma} g(y)\right) \omega_{\gamma} \psi_{\gamma}(y) d y
\end{aligned}
$$

where

$$
g(y)-T_{t}^{\gamma} g(y)=f(y)-T_{t}^{\gamma} f(y)+f(1)\left(T_{t}^{\gamma} q_{\gamma}(y)-q_{\gamma}(y)\right)
$$

It is a well-known property of the Wright-Fisher diffusion, derived using Itô's formula, that $q_{\gamma}\left(\xi_{t}\right)$ is a $P_{x}^{\gamma}$-martingale, in particular $E_{y}^{\gamma}\left[q_{\gamma}\left(\xi_{t}\right)\right]=q_{\gamma}(y)$.

Thus, $g(y)-T_{t}^{\gamma} g(y)=f(y)-T_{t}^{\gamma} f(y)$, and so

$$
N \mathbb{E}_{1 / N}^{\gamma}\left[\int_{0}^{t} f\left(\xi_{u}\right) d u\right] \rightarrow \int_{0}^{1}\left(f(y)-T_{t}^{\gamma} f(y)\right) \omega_{\gamma} \psi_{\gamma}(y) d y+f(1) \omega_{\gamma} t
$$

as required to show i). Statements ii) and iii) follow by observing

$$
\mathbb{E}_{\mathcal{X}_{0}}\left[\int_{[0,1] \times \mathcal{D}} f\left(\xi_{t}^{0, y}\right) \mathcal{M}_{\gamma}\left(d y, d \xi^{0, y}\right)\right]=\int_{0}^{1} T_{t}^{\gamma} f(y) \mathcal{N}_{\gamma}(d y)=\left\langle\mathcal{X}_{0}, T_{t}^{\gamma} f\right\rangle
$$

and

$$
\mathbb{E} \int_{0}^{1} T_{t}^{\gamma} f(y) \mathcal{N}_{\gamma}(d y)=\int_{0}^{1} T_{t}^{\gamma} f(y) \omega_{\gamma} \psi_{\gamma}(y) d y
$$

B. THE AFS DURING NONEQUILIBRIUM AFTER A

\section{CHANGE IN POPULATION SIZE}

The results in Appendix A extend to the $(\gamma, \kappa)$ Wright-Fisher model with infinitesimal generator

$$
Q_{\gamma, \kappa} f(x)=\gamma x(1-x) f^{\prime}(x)+\frac{1}{2 \kappa} x(1-x) f^{\prime \prime}(x), \quad f \in \mathcal{D},
$$


By Lemma 3 iii),

$$
\mathbb{E}\left\langle\mathcal{N}_{\gamma}, T_{t-t^{*}}^{\gamma, \kappa} f\right\rangle \rightarrow \int_{0}^{1} T_{t-t^{*}}^{\gamma, \kappa} f(y) \omega_{\gamma, 1} \psi_{\gamma, 1}(y) d y+T_{t-t^{*}}^{\gamma, \kappa} f(1) \omega_{\gamma, 1} t^{*}
$$


962

963

Since $T_{t}^{\gamma, \kappa} f(1)=f(1)$ for each $t$, it follows that

$$
\begin{aligned}
& \mathbb{E}\left\langle\mathcal{X}_{t}^{N_{\kappa}}, f\right\rangle \rightarrow \int_{0}^{1} T_{t-t^{*}}^{\gamma, \kappa} f(y) \omega_{\gamma, 1} \psi_{\gamma, 1}(y) d y+f(1) \omega_{\gamma, 1} t^{*} \\
& \quad+\int_{0}^{1}\left(f(y)-T_{t-t^{*}}^{\gamma, \kappa} f(y)\right) \omega_{\gamma, \kappa} \psi_{\gamma, \kappa}(y) d y+f(1) \omega_{\gamma, \kappa}\left(t-t^{*}\right)
\end{aligned}
$$

which is the desired relation and hence completes the proof.

C. NUCLEOTIDE DIVERSITY

For computing nucleotide diversity, we apply the AFS to the function $f_{\mathrm{pw}}(y)=2 y(1-y)$ and note that $f_{\mathrm{pw}}(1)=0$. For the neutral case it holds in equilibrium according to Eq. (7)

$$
\pi_{S}^{\kappa}=\theta \int_{0}^{1} 2 y(1-y) \omega_{0, \kappa} \psi_{0, \kappa}(y) d y=4 \theta \kappa \int_{0}^{1}(1-y) d y=2 \theta \kappa,
$$

since $\omega_{0, \kappa}=1$ and $\psi_{0, \kappa}=2 \kappa / y$. The synonymous diversity during nonequilibrium is obtained by applying the AFS in Eq. (10) to $f_{\mathrm{pw}}(y)$,

$$
\begin{aligned}
\pi_{S}^{\kappa}(t) & =\theta \int_{0}^{1} \mathbb{E}_{y}^{0, \kappa}\left[2 \xi_{t}^{t^{*}}\left(1-\xi_{t}^{t^{*}}\right)\right]\left(\frac{2}{y}-\frac{2 \kappa}{y}\right) d y+\theta \int_{0}^{1} 2 y(1-y) \frac{2 \kappa}{y} d y \\
& =2 \theta(1-\kappa) e^{-\left(t-t^{*}\right) / \kappa}+2 \theta \kappa
\end{aligned}
$$

where we used Itô's formula to compute $\mathbb{E}_{y}^{0, \kappa}\left[\xi_{t}^{t^{*}}\left(1-\xi_{t}^{t^{*}}\right)\right]=e^{-\left(t-t^{*}\right) / \kappa} y(1-y)$. It holds $\pi_{S}^{\kappa}\left(t^{*}\right)=2 \theta$ and $\pi_{S}^{\kappa}(t)=\pi_{S}^{\kappa}=2 \theta \kappa$ in the limit $t \rightarrow \infty$.

For nonsynonymous nucleotide differences we proceed similarly but use the DFE in Eq. (12) to allow for variation in selection. The stationary case follows by integration of Eq. (11) over the DFE,

$$
\mathbb{E} \pi_{N}^{\mathcal{V}, \kappa}=4 \theta \kappa \int_{-\infty}^{0}\left(\frac{1}{1-e^{-2 v \kappa}}-\frac{1}{2 v \kappa}\right) h_{\mathcal{V}}(v) d v
$$


This integral is well-defined, which can be seen by looking at the Taylor expansion of the integrand around zero and noting that the moments of the gamma distribution are finite. For the behavior of $\pi_{N}$ in nonequilibrium we apply the nonstationary AFS stated in Eq. (10) to $f_{\mathrm{pw}}(y)$, i.e.

$$
\begin{aligned}
\pi_{N}^{\kappa}(t)= & \theta \int_{-\infty}^{0} \int_{0}^{1} \mathbb{E}_{y}^{v, \kappa}\left[2 \xi_{t}^{t^{*}}\left(1-\xi_{t}^{t^{*}}\right)\right]\left(\omega_{v, 1} \psi_{v, 1}(y)-\omega_{v, \kappa} \psi_{v, \kappa}(y)\right) h_{\mathcal{V}}(v) d y d v \\
& +\theta \int_{-\infty}^{0} \int_{0}^{1} 2 y(1-y) \omega_{v, \kappa} \psi_{v, \kappa}(y) h_{\mathcal{V}}(v) d y d v \\
= & 2 \theta \mathbb{E} \int_{0}^{1} \mathbb{E}_{y}^{\mathcal{V}, \kappa}\left[\xi_{t}^{t^{*}}\left(1-\xi_{t}^{t^{*}}\right)\right]\left(\omega_{\mathcal{V}, 1} \psi_{\mathcal{V}, 1}(y)-\omega_{\mathcal{V}, \kappa} \psi_{\mathcal{V}, \kappa}(y)\right) d y+\mathbb{E} \pi_{N}^{\mathcal{V}, \kappa}
\end{aligned}
$$

Since $\xi_{t}^{t^{*}} \in\{0,1\}$ for $t \rightarrow \infty$, it holds $\mathbb{E}_{y}^{\mathcal{V}, \kappa}\left[\xi_{t}^{t^{*}}\left(1-\xi_{t}^{t^{*}}\right)\right]=0$ in the limit $t \rightarrow \infty$, implying $\pi_{N}^{\kappa}(t) \rightarrow \mathbb{E} \pi_{N}^{\mathcal{V}, \kappa}$. We can further explicitly write

$$
\begin{aligned}
& \omega_{\gamma, 1} \psi_{\gamma, 1}(y)-\omega_{\gamma, \kappa} \psi_{\gamma, \kappa}(y) \\
& =\frac{2}{y(1-y)}\left(\frac{1-e^{-2 \gamma(1-y)}}{1-e^{-2 \gamma}}-\frac{\kappa\left(1-e^{-2 \gamma \kappa(1-y)}\right)}{1-e^{-2 \gamma \kappa}}\right)
\end{aligned}
$$

for $\gamma \neq 0$, and $\psi_{0,1}(y)-\psi_{0, \kappa}(y)=2 / y-2 \kappa / y=2(1-\kappa) / y$.

D. THE NUMBER OF FIXATIONS

The number of fixations before the change in population size, i.e. $Z^{\gamma, \kappa}(t)$

for $t \leq t^{*}$, is obtained by applying the equilibrium AFS in Eq. (7) to $f_{\text {fix }}(y)$,

$$
Z^{\gamma, \kappa}(t)=\theta \omega_{\gamma, 1} f_{\text {fix }}(1) t+\theta \int_{0}^{1} f_{\text {fix }}(y) \omega_{\gamma, 1} \psi_{\gamma, 1}(y) d y=\theta \omega_{\gamma, 1} t
$$

The integral term vanishes and $\kappa=1$, since for $t \leq t^{*}$ the population size is $N_{\kappa}=N$ 
${ }_{987}$ For $t>t^{*}$, we apply the nonequilibrium AFS in Eq. (10),

$$
\begin{aligned}
Z^{\gamma, \kappa}(t)= & \theta\left\{\omega_{\gamma, 1} t^{*}+\omega_{\gamma, \kappa}\left(t-t^{*}\right)\right\} \\
& +\theta \int_{0}^{1} \mathbb{E}_{y}^{\gamma, \kappa} f_{\text {fix }}\left(\xi_{t}^{t^{*}}\right)\left(\omega_{\gamma, 1} \psi_{\gamma, 1}(y)-\omega_{\gamma, \kappa} \psi_{\gamma, \kappa}(y)\right) d y \\
& +\theta \int_{0}^{1} f_{\text {fix }}(y) \omega_{\gamma, \kappa} \psi_{\gamma, \kappa}(y) d y
\end{aligned}
$$

988 Since the last term vanishes and $\mathbb{E}_{y}^{\gamma, \kappa} f_{\text {fix }}\left(\xi_{t}^{t^{*}}\right)=\mathbb{P}_{y}^{\gamma, \kappa}\left(\tau_{1} \leq t-t^{*}\right)$, as we have 989 shown in Eq. (15), it follows

$$
\begin{aligned}
Z^{\gamma, \kappa}(t)=\theta & \left\{\omega_{\gamma, 1} t^{*}+\omega_{\gamma, \kappa}\left(t-t^{*}\right)\right\} \\
& +\theta \int_{0}^{1} \mathbb{P}_{y}^{\gamma, \kappa}\left(\tau_{1} \leq t-t^{*}\right)\left(\omega_{\gamma, 1} \psi_{\gamma, 1}(y)-\omega_{\gamma, \kappa} \psi_{\gamma, \kappa}(y)\right) d y
\end{aligned}
$$

${ }_{990}$ The explicit representation for $\omega_{\gamma, 1} \psi_{\gamma, 1}(y)-\omega_{\gamma, \kappa} \psi_{\gamma, \kappa}(y)$ is given in Eq. (C.1). 


\section{E. SUPPLEMENTARY FIGURES}

A

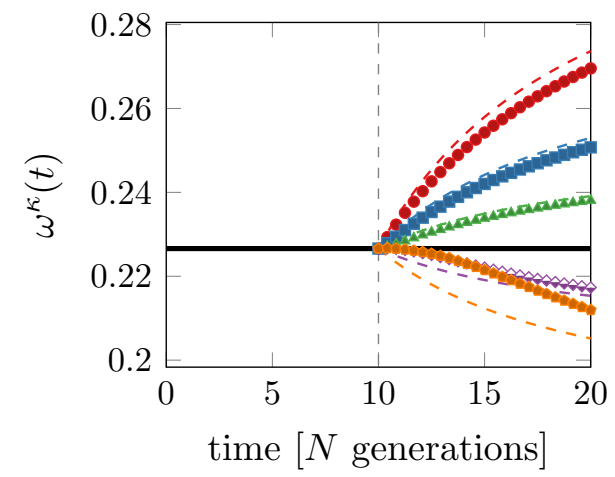

B

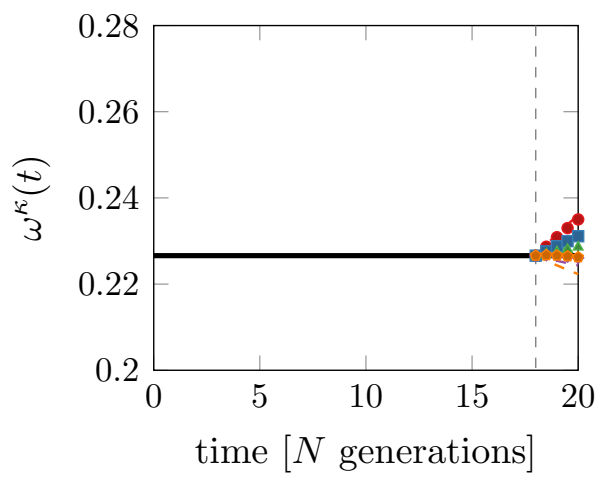

$$
\boldsymbol{\bullet} \kappa=0.1 \quad \varangle=0.25 \quad \Delta \kappa=0.5 \quad-\kappa=1 \quad \diamond \kappa=2 \quad \bullet \kappa=4
$$

Figure E.6: The fixation rate ratio $\omega^{\kappa}(t)$ for different values of $\kappa$ as functions of time. Panel A: change in population size at time $t^{*}=10$. Panel B: change in population size at time $t^{*}=18$. For comparison, colored, dashed curves represent the weighted fixation rate ratio $\omega_{\mathrm{w}}^{\kappa}(t)$. Vertical, dashed lines indicate the time $t^{*}$. Parameters: $\theta=1$, and $a=0.15$ and $a b=2500$ for the DFE. 


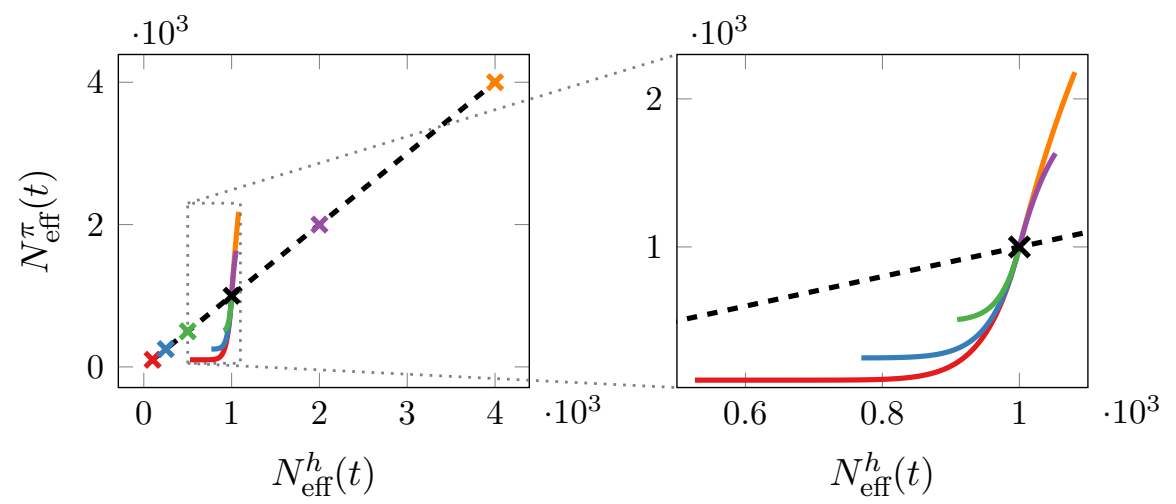

- - equilibrium $-\kappa=0.1-\kappa=0.25-\kappa=0.5-\kappa=2-\kappa=4$

Figure E.7: The effective population size based on nucleotide variation, $N_{\text {eff }}^{\pi}(t)$, versus the harmonic mean effective population size, $N_{\text {eff }}^{h}(t)$, as parametric curves of time after a recent change in population size, $t^{*}=18 \leq t \leq 20$, for different values of $\kappa$. Black, dashed lines show the expected behavior in equilibrium populations. Black crosses at $N_{\text {eff }}^{h}\left(t^{*}\right)=N_{\text {eff }}^{\pi}(t)=1000$ mark the time of change in population size, $t^{*}$. Colored crosses indicate the expected equilibrium value for each $\kappa$. Parameters $\theta=1$ and $N=1000$. 